\title{
Die Produktion eines „Problemviertels“‘. Mediale Diskurse, politisch-polizeiliche Interventionen und interaktive Situationsbedeutungen
}

\author{
Moritz Rinn · Jan Wehrheim
}

Angenommen: 23. Juli 2021 / Online publiziert: 31. August 2021

(C) Der/die Autor(en) 2021

Zusammenfassung Auf Grundlage qualitativ-ethnographischer Forschung und aus symbolisch-interaktionistischer Perspektive untersucht der Beitrag den wechselseitigen Verstärkungsprozess von negativen Stadtteilimages und polizeilichen Interventionen sowie dessen unterschiedliche Auswirkungen auf Alltage von Bewohner*innen problematisierter Stadtteile. Am Beispiel des seit mehreren Jahrzehnten stadtpolitisch als „,benachteiligt“ bearbeiteten Essen-Altendorf zeigt der Artikel auf, wie spezifische Images von ,gefährlichen“ oder ,problematischen“ Stadtteilen durch Wechselwirkungen zwischen medialer Berichterstattung, polizeilichen Interventionen sowie Situationsbe-deutungen von einzelnen Akteur*innen (re-)produziert werden. Es entstehen, so die Beobachtung, polizeilich-publizistische Verstärkerkreisläufe, die das „Problemviertel“-Image immer wieder neu reproduzieren und weitere, tendenziell restriktive polizeiliche und stadtpolitische Interventionen legitimieren. Neben „territorialer Stigmatisierung“ (Wacquant) der Bewohner*innen evozieren diese Interventionen konkurrierende Situationsdefinitionen, Problemkonstruktionen und Gegenerzählungen, die indes öffentlich selektiv wahrgenommen und bewertet werden. Stadtteilbewohner*innen greifen in ihren Situationsbe-deutungen auf unterschiedliche Wissens- bzw. Erfahrungsbestände zurück. Es zeigt sich, dass Perspektiven, die dem dominanten „Problemvierteldiskurs“ widersprechen - etwa indem sie ethnisierend diskriminierende polizeiliche Kontrollpraktiken thematisieren - im öffentlichen Diskurs großenteils marginalisiert bleiben.

\footnotetext{
Moritz Rinn $(\bowtie) \cdot$ Jan Wehrheim Institut für Soziale Arbeit und Sozialpolitik, Universität Duisburg-Essen, Campus Essen - Gebäude S06, Universitätsstraße 2, 45141 Essen, Deutschland

E-Mail: moritz.rinn@uni-due.de

Jan Wehrheim

E-Mail: jan.wehrheim@uni-due.de
} 
Schlüsselwörter Polizei · Stadtentwicklung · Territoriale Stigmatisierung · Stadtteilimages · Symbolischer Interaktionismus · Institutioneller Rassismus

\title{
The creation of a "troubled neighborhood": Media discourses, political interventions, policing and interactive definitions of situations
}

\begin{abstract}
Based on qualitative ethnographic research and following a symbolicinteractionist perspective, this paper examines both the relational process in which negative images of neighborhoods and police interventions reinforce each other and the way this affects the everyday life of residents. Focussing on Altendorf, a district treated as a disadvantaged area by the city government of Essen for decades, we show how images of "dangerous" or "troubled" neighborhoods are (re-)produced through interactions between media reporting, police interventions and situational interpretations by individual actors. These interactions create amplifying circuits that foster further, mostly restrictive policing and legitimize urban policy interventions. Such policies, however, do not only permanently reproduce the "territorial stigmatization" (Wacquant) of a district and its inhabitants but also evoke competing definitions of situations, perceptions of problems and counter-narratives, which then are selectively perceived and evaluated by the public. Yet, as they interpret situations unfolding in the district, residents draw on quite different discourses and experiences. As is shown, perspectives that contradict the dominant "troubled-areadiscourse", e.g. by mentioning repressive policing and racist controls or by explicitly rejecting Altendorf's image as a "dangerous" area, remain mostly marginalized in the public discourse.
\end{abstract}

Keywords Police - Urban development - Territorial stigmatization · Neighborhood image $\cdot$ Symbolic interactionism $\cdot$ Institutional racism

\section{La production d'un « quartier sensible » : Discours médiatiques, interventions politico-policières et interprétations interactives de situations}

Résumé Cet article étudie sur la base de recherches qualitatives ethnographiques inspirées de l'interactionnisme symbolique le processus de renforcement mutuel entre l'image négative de certains quartiers et les interventions policières dont il fait l'objet ainsi que les répercussions de ce processus sur le quotidien des habitant.e.s des quartiers désigné comme «sensibles ». À partir de l'exemple du quartier d'Altendorf à Essen qui fait l'objet depuis des décennies d'un traitement par les politiques urbaines comme « quartier défavorisé », cet article montre comment l'image de quartiers « dangereux » ou « sensibles » est reproduite par les interactions entre la couverture médiatique, les interventions policières et les interprétations de la situation par des acteurs particuliers. On observe que des boucles de renforcement politico-médiatiques reproduisent sans cesse l'image de «quartier sensible » et légitiment toujours plus d'interventions policières ou de politique urbaine tendanciellement restrictives. Outre la «stigmatisation territoriale » (Wacquant) des habitant.e.s, ces interventions provoquent des définitions de la situation, des constructions des problèmes et des 
" contre récits » qui sont cependant perçus et évalués de manière sélective dans l'espace public. Dans leurs interprétations de la situation les habitant.e.s des quartiers recourent à différents stocks de savoirs et d'expériences. Il apparaît que les perspectives qui contredisent le discours dominant du « quartier sensible »-par exemple en évoquant les pratiques de contrôle policier ethniquement discriminatoires restent en grande partie marginalisées dans le discours public.

Mots-clés Police · Développement urbain · Stigmatisation territoriale · Image des quartiers $\cdot$ Interactionnisme symbolique $\cdot$ Racisme institutionnel

\section{Einleitung}

Am 8. Dezember 2017 veröffentlichte die Westdeutsche Allgemeine Zeitung (WAZ) einen Artikel über einen Polizeieinsatz im Essener Stadtteil Altendorf (Kintscher 2017). ${ }^{1}$ Unter der Überschrift „Sicherheit. Nach der Randale: Polizei Essen will gezielt Stärke zeigen“ prangt ein Foto von acht Polizeiwagen, untertitelt mit „Mittwochabend in Altendorf: Viel Blaulicht um nichts. Doch die Polizei demonstriert so Stärke nach außen.“ Der Artikel beginnt fettgedruckt: „Dem Gefühl, dass sich in Essen etwas zusammenbraut und Paralleljustiz um sich greift, begegnet die Ordnungsmacht mit demonstrativer Präsenz". Erst im hinteren Teil des Artikels, der ohne Online-Abonnement nicht zugänglich ist, folgt die Aufklärung: Randale habe es in Altendorf nicht gegeben. Ein Stadtteilbewohner habe der Polizei Schüsse und die „Zusammenrottung“ von etwa zwanzig Männern gemeldet. Die alarmierten Ordnungskräfte seien nach diesem Anruf mit einem Großaufgebot angerückt, hätten jedoch vor Ort feststellen müssen, dass es sich bei den "Schüssen“ um laute Geräusche herunterfallender Metallplatten von dort tätigen Handwerkern und bei der „Zusammenrottung“ um Männer gehandelt haben musste, die sich anlässlich eines Fußball-Champions-League-Spiels von Borussia Dortmund vor einem Wettbüro aufgehalten hätten. Viel Blaulicht also um „nichts“?

Trotz der ,irrtümlichen“ Situationsdeutung des Anrufers ließ sich der Essener Polizeisprecher mit den Worten zitieren, es gehe darum, „Flagge zu zeigen, deutlich zu machen, dass wir in der Stadt keine Paralleljustiz dulden“. Mit Überschrift, Aufmacher und Bildmaterial bedient die Zeitung damit einen überlokalen Diskurs zu „Ghettoisierung“, „Parallelgesellschaften“ und jüngst „Clankriminalität“ und verortet diesen in Altendorf. Zudem werden indirekt Handlungsempfehlungen gegeben: Entsprechenden Gefahren im Kontext von insbesondere ethnischen Segregationsprozessen sei polizeilich mit „Stärke“ zu begegnen. Das Image des Stadtteils und seiner Bewohner*innen wird so durch den Zeitungsartikel und die polizeiliche Stellungnahme reproduziert - und damit auch das, was dort an Geschehnissen erwartbar ist.

\footnotetext{
${ }^{1}$ Die WAZ gehört zur Funke Mediengruppe und ist gemäß Selbstdarstellung die größte Regionalzeitung Deutschlands. Entsprechende Artikel erscheinen online identisch auch in der zum Unternehmen gehörenden Neuen Ruhr Zeitung, der Westfälischen Rundschau, dem Iserlohner Kreisanzeiger und Zeitung sowie der Westfalenpost. Die Druckauflage betrug Ende der 2010er Jahre zusammen noch über 500.000 Exemplare.
} 
Es ist zu vermuten, dass dieses Image die im Angesicht der späteren Aufklärung der Ereignisse irrational wirkende Situationsinterpretation des Anrufers und der Polizei angeleitet hatte: Stadtteilimage- oder raumproduzierende Diskurse (vgl. Glasze et al. 2005, S. 17 ff.) und Situationsbe-deutungen ${ }^{2}$ beeinflussen sich wechselseitig. Symbolische Repräsentationen haben deutliche materielle Effekte, die zugleich selektiv sind und vor allem die Alltagserfahrungen derjenigen prägen, die kulturalisierend bzw. ethnisierend als ,übliche Verdächtige“ etikettiert werden.

Im Folgenden sollen dieser wechselseitige Verstärkungsprozess und dessen unterschiedliche Auswirkungen auf die verschiedenen Quartiersalltage aus einer symbolisch-interaktionistischen Perspektive analysiert werden. Am Beispiel des seit mehreren Jahrzehnten stadtpolitisch als ,benachteiligt“ behandelten Stadtteils EssenAltendorf zeigen wir auf, wie spezifische Images von ,gefährlichen“ oder ,,problematischen" Stadtteilen in Wechselwirkungen zwischen medialer Berichterstattung, polizeilichen Interventionen sowie konkreten Situationsbe-deutungen von einzelnen Akteur*innen produziert und reproduziert werden. Es entstehen polizeilich-publizistische Verstärkerkreisläufe, die weitere, tendenziell restriktive polizeiliche und stadtpolitische Interventionen legitimieren (vgl. auch Wiest und Kirndörfer 2019, S. 15). Diese Kreisläufe reproduzieren jedoch nicht nur permanent die „Problematik“ und „Gefährlichkeit“ eines Stadtteils und damit auch die ,,territoriale Stigmatisierung“ (Wacquant 2018) der Bewohner*innen, sondern evozieren auch konkurrierende Situationsdefinitionen und Problemkonstruktionen - sprich: „counter-narratives“ (Garbin und Millington 2012, S. 2074). Diese sind wiederum unterschiedlich anschlussfähig an dominantere Diskurse sowie stadt- und kriminalpolitische Programme und werden dementsprechend selektiv öffentlich wahrgenommen und bewertet. Gleichzeitig wird sichtbar, dass Stadtteilbewohner*innen in ihren Definitionen beobachteter oder erfahrener Situationen auf durchaus unterschiedliche Wissensbzw. Erfahrungsbestände zurückgreifen und unterschiedlich in diesen Reproduktionsprozess involviert sind. Es zeigt sich, dass Bewohner*innen, die dem dominanten „Problemviertel“-Diskurs widersprechen, etwa indem sie repressive oder rassistisch diskriminierende polizeiliche Kontrollpraktiken thematisieren oder das Image Altendorfs als ,gefährlich“ und Ort von „Clankriminalität“ explizit zurückweisen, mit ihrer Perspektive zumeist marginalisiert bleiben.

Diese Befunde sind Ergebnisse der Analyse von empirischem Material, das wir im Rahmen des qualitativen Forschungsprojekts ,Aneignungskonflikte in mischungs-

\footnotetext{
2 Wir verwenden die Formulierung „,be-deuten“ im symbolisch interaktionistischen Sinn, um sichtbar zu machen, dass Bedeutungen aktiv ausgehandelt werden und damit auch soziale Wirklichkeit erst interaktiv hervorgebracht wird. Gleichermaßen verstehen wir „Probleme“ von Stadtteilen oder ihren Bewohner*innen nicht als objektiv vorliegend. Welche Phänomene oder auch Lebenslagen problematisiert werden, resultiert nicht aus den zugrundeliegenden Sachverhalten selbst (vgl. Schetsche 2014). In Interviews interessierten wir uns für Situationen, die die Interviewten als „schwierig“ deuten. „Kriminalität“ gehört dabei zu „Ärgernissen und Lebenskatastrophen“ (Hanak et al. 1989) wie andere potenziell schwierige Alltagssituationen auch. Nicht mögliche polizeilich-juristische Bewertungen solcher Situationen interessieren, sondern die Auswirkungen auf die Bewältigung des jeweiligen Alltags: „Was wir darstellen ist nicht ein verzerrtes Bild der ,Realität', sondern die Realität, welche die Menschen beschäftigt, die wir studiert haben, die Realität, die sie durch ihre Interpretationen ihrer Erfahrungen schaffen und in deren Sinne sie handeln“ (Becker 2014, S. 166).
} 
orientierten Stadtentwicklungsprozessen“ erhoben haben. ${ }^{3}$ Während mehrmonatiger Feldforschungen haben wir in Altendorf neben fokussierten ethnographischen Beobachtungen u.a. 80 teilstrukturierte Interviews mit 114 Bewohner*innen geführt und ethnographisch protokolliert. ${ }^{4}$ Diese Interviews zielten auf Narrationen selbst erlebter schwieriger Situationen und problematischer Ereignisse im Stadtteil. Zusätzlich führten wir weitere acht vertiefende und stärker biographisch orientierte narrative Interviews mit Bewohner*innen, die stark von potenziellen Verdrängungsprozessen und Marginalisierung bedroht sind. Für den vorliegenden Aufsatz haben wir die Erzählungen der interviewten Bewohner*innen sowie eigene Erhebungserfahrungen hinsichtlich der Thematisierung von Polizei und Polizeiarbeit im Stadtteil ausgewertet und mit Analysen politisch-administrativer, polizeilicher und medialer Dokumente sowie eigens durchgeführten Expert*inneninterviews mit professionellen Akteur*innen in Beziehung gesetzt.

Im Anschluss skizzieren wir zunächst knapp die stadtsoziologische Diagnose des „Problemquartiers“ und dessen Stigmatisierung in stadtentwicklungspolitischer Hinsicht (Abschnitt 2). Dabei legen wir einen Fokus auf den polizeilichen Umgang mit „gefährlichen“ städtischen Räumen und dessen selektive Auswirkungen auf die Bewohner*innen problematisierter Stadtteile. Vor diesem Hintergrund arbeiten wir daraufhin die spezifische stadtpolitische, polizeiliche und mediale Bearbeitung unseres Untersuchungsgebietes Essen-Altendorf heraus (Abschnitt 3). Anschließend rekonstruieren wir materialbezogen die Erzählungen von Bewohner*innen über Schwierigkeiten und Konflikte im Stadtteil, insbesondere mit Fokus auf die Akteurin Polizei

\footnotetext{
3 In dem noch laufenden, von der Deutschen Forschungsgemeinschaft finanzierten Projekt untersuchen wir zudem auch in Hamburg/Altona-Altstadt potenziell konflikthafte Situationen im Zusammenhang mit Politiken sozialer Mischung und Gentrification. Beide Stadtteile sind bzw. waren als ,Stadtteile mit besonderem Entwicklungsbedarf" klassifiziert. Der auch kriminologische Schwerpunkt dieses Aufsatzes ist Folge dessen, dass Interviewte zunächst von sich aus die Polizei als eine maßgebliche Stadtteil-Akteurin in die Interviews einbrachten. An den Erhebungen in Altendorf waren neben den Autoren Fatemeh Afshar, Margarete Killian, Lena Wiese und Clara Will beteiligt.

${ }^{4}$ Um unterschiedliche Erfahrungen und Perspektiven auf verschiedene Konfliktfelder im Stadtteil zu erheben, strebten wir ein möglichst heterogenes Sample an Interviewpartner*innen an. Deshalb führten wir die meisten Interviews wochentags zu unterschiedlichen Zeiten nach Ansprachen im öffentlichen Raum (an Straßenecken, Plätzen und Parks) unmittelbar vor Ort. Wir verzichteten auf Audioaufzeichnungen und damit auch auf die Erhebung personenbezogener Daten, um die notwendigen ,Formalitäten“ (einer EU-DGSVO-konformen mehrseitigen schriftlichen Einverständniserklärung) und damit verbundene Ablehnungen zu minimieren, die sich mutmaßlich negativ auf die Heterogenität des Samples ausgewirkt hätten. Von den Interviewpartner*innen haben wir 54 als weiblich, 59 als männlich kategorisiert, knapp die Hälfte als zwischen 31 und 60 Jahre alt (sowie 23 Personen als über 60 Jahre und 11 Personen als 18 Jahre und jünger). Etwas mehr als der Hälfte (61 Personen) der Interviewten wurde von uns kein Migrationshintergrund zugeschrieben, den anderen ein Migrationshintergrund und/oder nicht-deutsche Staatsangehörigkeit. Dabei ging es nicht um eine „exakte“ Einordnung, sondern darum, die Zuschreibungen der Interviewer*innen mit zu erheben und auch für die weitere Auswertung festzuhalten, ob wir Erfahrungen der Interviewten mit rassifizierenden Zuschreibungen annehmen können, auch wenn sie von diesen möglicherweise nicht explizit berichten. Die Länge der Interviews variierte deutlich: Zwischen nur wenigen Minuten und fast zweieinhalb Stunden. Auf einen offenen Einstieg folgten thematische Nachfragen nach unterschiedlichen städtischen Ressourcen wie Wohnraum, sozialen und kulturellen Stadtteilinfrastrukturen und öffentlichen Räumen. Die Interviewprotokolle wurden anschließend im Team aus einer interaktionistischen Perspektive verstehend interpretiert (vgl. grundsätzlich Steinert 1998; Bareis und Cremer-Schäfer 2013). Es wurden knapp 150 Erzählungen über selbst erlebte schwierige Situationen sowie 180 eher allgemein bleibende Erzählungen über Probleme im Stadtteil rekonstruiert.
} 
(Abschnitt 4). Dabei arbeiten wir zudem unterschiedliche Strategien des Umgangs mit dem negativen Stadtteilimage, Zuschreibungen von Kriminalität sowie beobachteten oder selbst erfahrenen Polizeikontrollen heraus. Schließlich zeigen wir am Beispiel zweier unsererseits beobachteter und medial verhandelter Kontrollsituationen, wie Interaktionsdynamiken territorialer Stigmatisierung in actu „funktionieren“ und sich durch diese hindurch Bedeutungshierarchien herstellen (Abschnitt 5).

\section{Territoriale Stigmatisierung und die Polizei als ,primary definer“}

Residentielle Segregation beschäftigt die stadtsoziologische Forschung seit den frühen Arbeiten der Chicago School der 1920er-Jahre. Fristete das Thema in der deutschsprachigen Stadtsoziologie der Nachkriegszeit lange ein Nischendasein, so werden Prozesse urbaner Marginalisierung und damit verknüpfte „Quartierseffekte“ seit den 1990er-Jahren auch hierzulande breit erforscht und diskutiert (vgl. seinerzeit Dangschat 1997). Im Zentrum steht dabei unter anderem die Frage, inwieweit die städtische Segregation sozial unterprivilegierter und ethnisierter Personengruppen sich negativ auf deren Chancen auf soziale Teilhabe auswirkt - zusätzlich zu ihrer ohnehin meist prekären sozialen Lage. Immer wieder wurden und werden von soziologischer Seite auch die politischen Gefahren von Segregationsprozessen für die städtische respektive gesamtgesellschaftliche Kohäsion problematisiert (vgl. u.a. Heitmeyer 1998; Häußermann und Kronauer 2009). ${ }^{5}$

Neben sozialräumlich variierenden Chancen auf Bildungs- und Arbeitsmärkten betonen Stadtsoziolog*innen seit längerem auch die symbolische Dimension urbaner Marginalisierung und damit die Auswirkungen von „Quartiersimages“. Ganze Stadtteile mitsamt ihrer Bewohner*innenschaft würden stigmatisiert, was sich wiederum indirekt nicht nur auf deren Teilhabechancen, sondern auch auf Repräsentationen und Identität auswirke. So beschrieb Loïc Wacquant (1996) das Ineinandergreifen von Marginalisierung und Stigmatisierung in seinem prominenten Vergleich des Viertels Woodlawn in der Chicagoer Southside und der Großsiedlung Quatre Mille im Pariser Vorort La Courneuve. Trotz betonter Unterschiede zwischen französischen und US-amerikanischen Städten spricht er dabei in Bezug auf Bourdieus (1998a, S. 21) Konzept der ,symbolischen Gewalt“ und Goffmans Analysen des „Stigmamanagements“ jeweils von ,territorialer Stigmatisierung“ (Wacquant 2018, S. $180 \mathrm{ff}$.). Bewohner*innen verlören nicht nur die Deutungshoheit über ihre Repräsentation und müssten ihre ,,beschädigte Identität“ (Goffman 1967) managen: Territoriale Stigmatisierung zerstöre gar nachhaltig die sozialen Beziehungen und Netzwerke innerhalb der heterogenen Bevölkerung verunglimpfter Orte und raube den Bewohner*innen nicht zuletzt die Fähigkeit zu kollektivem Handeln, also auch

\footnotetext{
5 Die Identifikation ,,benachteiligter Stadtteile“ war und ist dabei in der deutschen Stadtsoziologie häufig durchaus mit einem kritischen Impetus gegen auch symbolische „,soziale Ausgrenzung“ verknüpft. Gleichzeitig, so zeigt etwa Christiane Reinecke (2021) in ihrer zeithistorischen Arbeit zu ,urbanen Problemzonen“, hat ihr problematisierender Blick auf die ,,Verräumlichung des Sozialen“ die diskursive Konstruktion von „Problemquartieren“ vorbereitet und mitgestaltet, mitsamt stigmatisierender Effekte kulturalistisch und rassistisch gefärbter Debatten um ,neue Unterschichten“ und „Parallelgesellschaften“ auf die dortigen Bewohner*innen.
} 
zur kollektiven Artikulation von Gegendiskursen (vgl. Wacquant 2018, S. XX ff.). Während Wacquant diese These in späteren Arbeiten differenzierte (Wacquant et al. 2014, S. 1276), verwiesen Autoren wie Garbin und Millington (2012) im Anschluss an seine Ausgangsbeobachtungen auf vielfältige Formen widerständigen Umgangs mit Marginalisierung und Stigmatisierung.

Die jüngere Forschung zu Segregation in deutschen Großstädten betont nicht nur die deutlichen Unterschiede im Vergleich zu US-amerikanischen oder französischen urbanen Verhältnissen, sondern auch den Mangel an empirischen Belegen für übergreifende „Quartierseffekte“ (vgl. Walther und Güntner 2013; Volkmann 2012). Zurückgeführt wird dies unter anderem auf eine im Vergleich zu Frankreich und vor allem den USA hohe sozioökonomische und soziokulturelle Heterogenität der problematisierten Stadtteile sowie auf deutliche Unterschiede in Bezug auf die objektivierte soziale Lage der Bewohner*innen oder auch auf als gewalthaft gedeutete Ereignisse. Stigmatisierung und Images als „Problemviertel“ und ,gefährliche Viertel“ erfahren gleichwohl zunehmende Aufmerksamkeit in der Stadtforschung, wobei zum einen auf hegemoniale negative „Quartiersimages“ verwiesen wird, zum anderen auf durchaus differenzierte Binnenwahrnehmungen durch Bewohner*innen, die von diesen Images auf unterschiedliche Art und Weise betroffen sind und sich entsprechend verschieden zu ihnen positionieren (vgl. Gestring 2012; Boller 2013).

Das länderübergreifend gemeinsame Fundament territorialer Stigmatisierung, der sich Bewohner*innen ausgesetzt sehen, bildet der seit den Urbanisierungs- und Industrialisierungsprozessen des 19. Jahrhunderts bestehende diskursive Nexus von Armut, moralischem Verfall und Kriminalität, der das stereotype Bild der segregiert lebenden ,gefährlichen Klassen“ grundiert (vgl. Morris 1994; Lindner 2004; Wehrheim 2012). Zentrale Elemente dessen sind dabei auch im deutschen Fall seit jeher ethnisierende und rassifizierende Zuschreibungen von Devianz, Kriminalität und Unsicherheit, die in der Rede von „Parallelgesellschaften“, „Ghettoisierung“ und „No-go-Areas“ zum Ausdruck kommen (vgl. Ronneberger und Tsianos 2009; Wiest und Kirndörfer 2019; Mohring und Rolfes 2020, Reinecke 2021) sowie in den entsprechenden sozial-, aber gerade auch kriminal- und ordnungspolitischen Interventionen ihren Niederschlag finden.

Vermutlich am deutlichsten zeigt sich Letzteres auf der Ebene von Stadtteilen in der Definition sogenannter „Gefahrengebiete“ gemäß Polizeirecht (vgl. Keitzel 2020) sowie in einem entsprechend durch Null-Toleranz-Politiken und „Broken Windows“-Argumentationen legitimierten autoritären raumbezogenen ,Polizieren“ (vgl. Belina und Wehrheim 2011; Wehrheim 2012). Damit assoziierte selektive polizeiliche Maßnahmen wie das „Racial Profiling“ werden seit jüngstem verstärkt auch in Deutschland in intersektionaler Perspektive diskutiert, etwa hinsichtlich der klassenbezogenen Selektivität von Praktiken des ,,policing“ (vgl. Belina 2018; Thompson 2018; Künkel 2014). Doch auch in der klassischen Polizeiforschung wird selektiven Kontrollen und „Entscheidungsspielräumen“ der Polizei wieder verstärkte Aufmerksamkeit geschenkt, gerade mit Blick auf spezifisch problematisierte Örtlichkeiten und Wohnquartiere (vgl. z.B. Ruch 2017).

Befunde zu „Racial Profiling“ sind inzwischen international gut dokumentiert (vgl. u.a. Glaser 2015; Waddington et al. 2004; Thompson 2018). Kriminologische Forschung hat gezeigt, dass Diskriminierung sich auch über die sozialräumliche 
Selektivität entsprechender polizeilicher Praktiken und institutioneller Maßnahmen manifestiert (vgl. für US-amerikanische Städte z.B. Terrill und Reisig 2003; für deutsche Städte z.B. Hunold 2015; für französische Städte z.B. Fassin 2018). Der Anteil nicht-weißer Menschen und People of Color an der Stadtteilbevölkerung, der Grad ,ethnischer“ Heterogenität, die relative personelle Ausstattung der Polizeireviere sowie die relative sozialstrukturelle Lage der Bewohner*innen der Stadtteile gelten dabei als sozialräumliche Einflussfaktoren. „Based on a set of internalized expectations derived from past experience“, so beobachtete Smith schon vor vielen Jahren, unterteile die Polizei ,the population and the physical territory they must patrol into readily understandable categories“. Daraus resultiere ,a process of ecological contamination in which all persons encountered in bad neighborhoods are viewed as possessing the moral liability of the area itself" (Smith 1986, S. 316, Herv. d. A.).

Jüngere Forschungen zeigen jedoch, dass nicht die Nachbarschaft an sich, sondern die Verbindung der polizeilichen Typisierung von in der Regel männlichen Tätern mit Vorstellungen von Normen und Normalitäten innerhalb spezifischer Straßenzüge und Wohngebiete - also das bei Polizist*innen vorherrschende Image der Stadtteile - entscheidend ist für das polizeiliche Vorgehen (vgl. Belina und Wehrheim 2011; Hunold 2015, S. 163 ff.). Damit zeigt sich die ,ecological contamination“ als Ausdruck und Folge territorialer Stigmatisierung und der Definition von Gefahrengebieten als ihrer entsprechenden Institutionalisierung. Dass die heterogene Bewohner*innenschaft von der polizeilichen Praxis vor Ort dennoch auf unterschiedliche Art betroffen ist, führt innerhalb ihrer wiederum zu divergierenden Interpretationen und beeinflusst sowohl die jeweilige Deutung lokaler Ereignisse als auch ihre Beziehungen untereinander.

Mohring und Rolfes (2020) haben in ihrer Forschung zu „No-go-Area“-Diskursen und negativen Stadtteilimages in Deutschland unlängst die Bedeutung von Wechselwirkungen zwischen Medien und Politik für die Imagekonstruktion betont. Unserer Einschätzung nach muss jedoch gerade hinsichtlich Devianz und Kriminalität als „klassischem“ Kern von Problemimages auch der Polizei eine hohe Relevanz zugeschrieben werden. Sie nämlich fungiert als die zentrale Institution für die öffentliche und vorjuristische Definition von Kriminalität. Ihr proaktives Agieren und ihre institutionelle Funktion als Adressatinnen für Anzeigen und Beschwerden lässt städtischen Polizeien eine maßgebliche Bedeutung für die Ausprägung öffentlicher Vorstellungen über die „Gefährlichkeit“ von Stadtteilen und daran anschließende Stigmatisierungen zukommen. Ihr Einfluss ist dabei ein doppelter.

Zum einen deuten Polizist*innen inkriminierte konkrete Situationen als mehr oder weniger relevant hinsichtlich ihrer zeitlich und personell begrenzten Ressourcen und Aufgaben. Devianz und Kriminalität liegen dabei nicht dinghaft vor, sondern sind das Produkt interaktiver Zuschreibungen und Bewertungen von Handlungen. Wie sonstige soziale Akteure interpretieren auch Polizist*innen „Dinge“ im Sinne Herbert Blumers (1981) innerhalb der Kontexte, innerhalb derer sie wahrgenommen werden. ${ }^{6}$ Dabei kommt ihnen, wie Feest und Blankenburg (1972) verdeutlichten,

\footnotetext{
${ }^{6}$ Die symbolisch-interaktionistisch informierte Soziologie und Kriminologie hat vielfach darauf hingewiesen, dass dies über Ermessensspielräume der Polizei hinausgeht (vgl. Sack 1968; Peters 2009).
} 
eine besondere „Definitionsmacht“ zu: Polizist*innen verfügen über die Macht, ihre Situationsdefinitionen durchzusetzen. Zugleich ist die „Beschwerdemacht“ (ebd.) betroffener Personen, also die Macht eigene Realitätsdefinitionen gegen die polizeiliche einzubringen, sozial ungleich verteilt.

Zum anderen äußert sich der Einfluss der Polizei in der medienöffentlichen Repräsentation des Geschehens. So zeigten Stuart Hall et al. in ihrer Studie Policing the crisis, dass Polizeien als ,primary definers“ (Hall et al. 1978, S. $57 \mathrm{ff}$.) nicht nur meist als erste, sondern auch als anerkannte Expert*innen für Kriminalitätsphänomene auftreten und somit den Interpretations- und Problematisierungsrahmen für spezifische Ereignisse setzen (ebd., S. 69). Nachfolgende Definitionen bzw. Interpretationen der Ereignisse durch andere beteiligte oder nicht beteiligte Akteur*innen müssen sich daher auf diese polizeiliche Primärdefinition beziehen. Dies verweist auf die in hierarchisch strukturierten Gesellschaften bestehende ,Hierarchie der Glaubwürdigkeit" (Becker 2016 [1967], S. 12) und damit den Umstand, dass die sozial ungleiche Verteilung von Definitions- und Beschwerdemacht über die Ausgangssituationen hinausweist. Perspektiven und Realitätsdefinitionen Subalterner oder gar als deviant respektive kriminell etikettierter Individuen finden nicht nur in juristischen Kontexten weniger Gehör, sondern erhalten auch in der medialen Repräsentation vergleichsweise wenig Aufmerksamkeit.

Öffentliche bzw. mediale Stellungnahmen von involvierten Polizist*innen und polizeilichen Pressesprecher*innen, von Polizeigewerkschaften oder auch aus Innenministerien zu konkreten Ergebnissen, generellen Praktiken oder Arbeitsschwerpunkten können also nicht als objektive Berichte gelten, sondern müssen als perspektivische Interpretationen und zumindest implizit auch als (professions-)politische Äußerungen gelesen werden. Sie sind Teil der ,symbolische[n] Seite der Politik“ (Edelman 1976, S. 1, Hervorh. i. Orig.). Hall et al. (1978, S. 59 ff.) zufolge sind Medien erst sekundäre Definitionsinstanzen, die bei der Produktion und Verbreitung von Wissen zum typischen Themenkomplex „Kriminalität“ auf die „Expertise“ der Polizeien und deren Primärdefinitionen zurückgreifen. Auch eigene politische Agenden professioneller medialer Akteur*innen können hinsichtlich „Kriminalität“ nicht unabhängig von polizeilichen Deutungen erfolgen. ${ }^{7}$

Polizeiliche Deutungen und daran anschließende Aktivitäten mit Bezug auf Stadtteile und ihre Images haben also eine doppelte, in der Stadtsoziologie in dieser Verbindung bislang kaum berücksichtige Relevanz: Sie prägen einerseits die Definitionen und Bewertungen von Sachverhalten, die Gegenstände unmittelbaren polizeilichen Handelns sind (z.B. Deutungen als Ruhestörung, Körperverletzung, Widerstand); andererseits präformieren sie sowohl die öffentliche Darstellung dieser Sachverhalte als auch wiederum das polizeiliche Verhalten dazu. Wie diese doppel-

\footnotetext{
7 Im Kontext neuer Kommunikationstechnologien wird die doppelte Definitionsmacht der Polizei neu und machtvoll verhandelt: Einerseits werden Videos polizeilicher Aktivitäten wie etwa der Tötung von George Floyd oder, weniger bekannt, von Adel B. aus dem im Folgenden behandelten Stadtteil Essen-Altendorf vermehrt bei YouTube, Twitter, Instagram veröffentlicht. Andererseits nutzen Polizeien dieselben neuen Medien, stellen sich und Ereignisse dar und nutzen teilweise ihre Definitionsmacht, um unmittelbar weiteres Geschehen und Deutungen zu beeinflussen (vgl. zu Twitteraktivitäten der Polizei: Bröckling 2018). Siehe auch die Diskussion über die polizeilichen Stellungnahmen zu den Ereignissen Silvester 2019/20 in Leipzig (vgl. Wegner et al. 2020).
} 
te Definitionsmacht im Polizieren und im Diskurs über ein „,benachteiligtes Quartier“ zum einen konkret wirkmächtig wird und wie Bewohner*innen zum anderen mit selektiver Polizeiarbeit als Element territorialer Stigmatisierung umgehen, untersuchen wir nun im Folgenden am empirischen Beispiel Essen-Altendorf.

\section{Essen-Altendorf als Gegenstand von Stadtentwicklungs- und Kriminalpolitik und medienöffentlichen Problematisierungen}

Altendorf unterscheidet sich deutlich von den bei Wacquant und anderen $\mathrm{Au}$ tor*innen thematisierten Stadtteilen. Weder liegt dieses Essener Quartier an der Peripherie fernab von Arbeitsplätzen und städtischer Infrastruktur - Innenstadt und Hauptbahnhof sind in knapp 15 Minuten per ÖPNV oder Rad zu erreichen -, noch gibt es dort massenhaft verfallene oder leerstehende Häuser und Geschäfte. Auch in der Polizeilichen Kriminalstatistik ist Altendorf im Städte- und innerstädtischen Stadtteilvergleich unauffällig (vgl. DHPol 2020). Lediglich bei Phänomenen, die direkt auf Polizeipräsenz selbst zurückgehen, also Kontrolldelikten wie Verstößen gegen das Betäubungsmittelgesetz oder „Respektlosigkeiten Jugendlicher gegenüber der Polizei“ (Hagenbucher 2017) ist Altendorf, wie der Essener Polizeipräsident auf einer von uns besuchten Stadtteilveranstaltung bemerkte, auffällig (vgl. auch DHPol 2020, S. 24).

Wenngleich dem Stadtteil damit vermeintliche Hauptmerkmale eines „Problemviertels“ fehlen, kann er mit Blick auf Diskussionen über Segregation, Stadtentwicklung und territoriale Stigmatisierung als beispielhaft angesehen werden. So gilt Altendorf als benachteiligter und zugleich benachteiligender Stadtteil ,mit besonderem Erneuerungsbedarf“ (Stadt Essen 1999, S. 8). Die zahlreichen städtebaulichen, sozialarbeiterischen sowie polizeilichen Maßnahmen hatten jedoch bislang nicht den politisch erhofften desegregierenden und ,,aufwertenden“ Effekt: Auch 20 Jahre nach Aufnahme in das Programm ,Soziale Stadt“" ist der Anteil derer, die staatliche Transferleistungen beziehen, mehr als doppelt so hoch wie im Essener Durchschnitt (Leistungen nach SGB II: 32,1\% zu 15\%, vgl. Stadt Essen 2020). Ähnliches gilt für den zweiten typischen Sozialindikator, die Anzahl von Einwohner*innen mit nicht-deutscher oder doppelter Staatsangehörigkeit (49,9\% zu 27,2\%, ebd.). Auch wenn solche Angaben für sich nichts über die Qualität der sozialen Beziehungen im Stadtteil aussagen, so gelten die Zahlen als Indikatoren für Probleme - was sich mit Ronneberger und Tsianos (2009) als Hinweis auf die Institutionalisierung und eigenständige Bedeutung ethno-nationalistischer Wissensrepertoires in Prozessen territorialer Stigmatisierung verstehen lässt.

Zwei Mitarbeiter*innen der Stadtverwaltung sehen im Interview die Ursache der sozialen Zusammensetzung im lokalen Wohnungsmarkt begründet (Exp_Adorf_04). Das Mietniveau in Altendorf sei zu gering, für Eigentümer*innen gebe es kaum Anreize, in ihre Wohnungen zu investieren und diese aufzuwerten, auch weil es angesichts des anhaltenden Imageproblems an zahlungskräftiger Nachfrage mangele, und so sei der Stadtteil (erneut) zu einem „Ankommensstadtteil“" geworden. Infolgedessen nutzten zu viele von den Mitarbeiter*innen als kulturell ,,anders“ beschriebene Menschen, insbesondere aus „Südosteuropa“, den öffentlichen Raum. 
Konflikte zwischen verschiedenen ethnisierten Gruppen und damit Ordnungsprobleme seien, so betonen auch zwei weitere interviewte Mitarbeiter*innen der Quartiersentwicklung (Exp_Adorf_01), ebenso die Folge wie der Wegzug der eigentlich erwünschten Bewohner*innen, also auch derjenigen, die sich für den Stadtteil engagierten. Bauliche Leuchtturmprojekte zur Aufwertung des Stadtteils wie die Erneuerung zentraler Stadtplätze und die Errichtung eines höherpreisigen Neubauquartiers mit angrenzender künstlicher Wasserfläche - das sogenannte „Uferviertel“ am Niederfeldsee - werden als Strategien benannt, um die Attraktivität des Stadtteils $\mathrm{zu}$ verbessern, Investitionen in den Wohnungsbestand anzureizen und die Bevölkerungszusammensetzung zu verändern. Gewünscht werden mehr Mittelschichtshaushalte, insbesondere Familien, weniger Zuzug der „Problematischen“ und weniger Wegzug der „Erfolgreichen“ und „Engagierten“ (die es im Stadtteil auch gebe), wobei hier rassifizierende und klassenbezogene Differenzmarker ineinandergreifen. Die entsprechenden Politiken sozialer Mischung (vgl. bspw. Münch 2014; Bridge et al. 2012) werden dabei ergänzt durch Verhaltenspolitiken zur Kriminalprävention wie etwa Mitternachtsbasketball für Jugendliche oder Initiativen zur Müllbeseitigung.

Auffällig ist, dass sowohl in den Expert*inneninterviews als auch in offiziellen Publikationen der Stadt zwar der Mangel an positiven Erzählungen über den Stadtteil beklagt wird. Gleichzeitig wird die Problemerzählung jedoch in Form der klassischen Unterscheidung von würdigen und unwürdigen sowie gefährlichen Armen (vgl. z.B. Morris 1994) aufrechterhalten und katalysiert, indem spezifische Personengruppen pauschal - etwa über sozialarbeiterische Projekte - problematisiert werden (vgl. ausführlicher Rinn und Wiese 2020). Insofern zeigen sich bei der „linken Hand des Staates" (Bourdieu 1998b) die Ambivalenzen und Paradoxien einer sozialdemokratischen Stadtteilentwicklung und Sicherheitsorientierung: Die involvierten Institutionen schaffen sich neue Arbeitsfelder und generieren Finanzierungen, doch ihre Maßnahmen zur „Aufwertung“ und „Durchmischung“ befördern das Problemund Kriminalitätsimage des Stadtteils zugleich weiter - und zwar unabhängig von deren Erfolg und sonstigen Effekten (vgl. auch Baum und Otto 2020).

Bei der „rechten Hand des Staates“ (Bourdieu 1998b) lassen sich zunächst für die vergangenen knapp zehn Jahre intensivierte polizeilich-politische Interventionen in vier Bereichen in Altendorf beobachten. Dazu gehört erstens die privilegierte Problematisierung und Bearbeitung von Ordnungswidrigkeiten. In deren Hintergrund stehen die im Kontext des Problemviertel-Diskurses virulenten, aber empirisch nach wie vor nicht bestätigten Thesen der „Broken Windows“-Theorie, derzufolge nicht sanktionierte substrafrechtliche ,social and physical disorder“-Erscheinungen zu Kriminalitätsfurcht, Abnahme informeller sozialer Kontrolle, letztendlich ,richtiger" Kriminalität und damit zum weiteren Niedergang von Nachbarschaften führen (vgl. Wilson und Kelling 1982). Damit verbunden erfolgte zweitens, so ein von uns interviewter Polizeibeamter, ab dem Jahr 2015 der Einsatz einer lokalen ,TaskForce“ (Exp_Adorf_05) zur Bekämpfung insbesondere des Betäubungsmittelhandels in Altendorf, die im Zusammenhang mit der Verdrängung von „Drogenszenen“ an anderen Orten in Essen nach Altendorf zu sehen ist - die aber auch allgemein dazu dienen sollte, ,wieder einen Fuß darein“, d.h. in den Stadtteil, zu bekommen, der zu einer polizeilichen „No-go-Area“ zu werden gedroht habe (ebd.). Drittens wurden weitreichende Teile Altendorfs gemäß $§ 12$ des Polizeigesetzes des Landes 
Nordrhein-Westfalen zum „Gefahrengebiet“ erklärt und somit räumlich begrenzte verdachtsunabhängige Kontrollen im Stadtteil legalisiert (vgl. Landtag NordrheinWestfalen 2020). ${ }^{8}$ Viertens schließlich propagierte das nordrhein-westfälische Innenministerium ab 2018 die „Strategie der 1000 Nadelstiche“ gegen die sogenannte „Clan-Kriminalität“, was sowohl der Essener Oberbürgermeister Thomas Kufen (CDU) als auch Polizeipräsident Frank Richter öffentlich goutierten.

Die öffentliche Legitimation dieser Strategie funktioniert dabei etwa wie folgt: Weil die Clanstrukturen so unübersichtlich seien, müssten polizeiliche Zugriffe extensiv erfolgen, von Falschparken und Verstößen gegen Hygienevorschriften in Imbissen, über Schlägereien und den Besitz unversteuerten Tabaks bis hin zu Insolvenzverschleppung und Sozialleistungsbetrug. Die sicht- und berichtbaren Interventionen erfolgen somit nicht nur polizei- und strafrechtlich, sondern auch durch die Mitarbeit von Zoll, Steuerfahndung oder Gesundheitsämtern (vgl. Maibaum 2019a, 2019b; Böhme 2019). Die Unbestimmtheit bei der „Clanzugehörigkeit ${ }^{“ 9}$ und fehlende Möglichkeiten, den allgemeinen Kriminalitätsvorwurf in personenbezogener Strafverfolgung zu konkretisieren, ${ }^{10}$ legitimieren dabei einen repressiven Zugriff auf scheinbar beliebige Personen in Form polizeilicher Kontrollen im öffentlichen Raum und bei Razzien. Die Identifikation Altendorfs als Wohn- und Betätigungsort der „Clans“ verschärft den ohnehin in polizeilichen Nulltoleranzstrategien angelegten Generalverdacht gegen als migrantisch gedeutete Personen. Verdachtsunabhängige Kontrollen im Gefahrengebiet führen bereits aufgrund der soziodemographischen Struktur des Stadtteils dazu, dass nicht weiß-deutsch aussehende Bewohner*innen häufiger von Kontrollen betroffen sind als es ihrer bundesweiten statistischen Repräsentanz entspricht (vgl. auch Belina und Wehrheim 2011). In seiner doppelten Unbestimmtheit befördert das „Anti-Clan-Policing“ diese Tendenz weiter und markiert Mitarbeiter*innen und Besucher*innen von Imbissen, Friseursalons, ShishaBars und anderen, alltäglich genutzten Einrichtungen im Stadtteil stereotyp als Teile organisierter Kriminalität. Unabhängig von eventuellen ermittlungstaktischen Erfolgen wird im Zusammenspiel solcher polizeilicher Praxen institutioneller Rassismus wirksam.

\footnotetext{
${ }^{8}$ Das 2019 novellierte Polizeigesetz ermöglicht seitdem die „Strategische Fahndung“( $\$ 12$ a PolG NRW), die anlass- und ortsbezogen verdachtsunabhängige Kontrollen legalisiert.

9 Der Definition des Landeskriminalamtes zufolge umfasst der Begriff „Clankriminalität“ die „,vom Gewinn- oder Machtstreben bestimmte Begehung von Straftaten unter Beteiligung Mehrerer, wobei in die Tatbegehung bewusst die gemeinsame familiäre oder ethnische Herkunft als verbindende, die Tatbegehung fördernde oder die Aufklärung der Tat hindernde Komponente einbezogen wird, die Tatbegehung von einer fehlenden Akzeptanz der deutschen Rechts- oder Werteordnung geprägt ist und die Straftaten einzeln oder in ihrer Gesamtheit von erheblicher Bedeutung sind." (LKA NRW 2019, S. 7). Die auffallende Unschärfe dieser Definition legt nahe, dass diese „Definition“ vor allem der Interventionslegitimierung dient: Die fünf „oder“ ermöglichen einen breiten Interpretationsspielraum. Dadurch trifft die „Definition“ auch auf die deutsche Automobilindustrie mit ihren systematischen Abgasmanipulationen mit - um es dramatisierend zu formulieren - letztendlich tödlichen Konsequenzen, auf Banken mit Cum-Ex-Geschäften oder bekannte „Familienunternehmen“ wie Schlecker oder Merck zu. Vgl. zur statistischen Erfassung von „Clan-Kriminalität“" auch den investigativjournalistischen Artikel von Amjahid (2020).

${ }^{10}$ Im LKA-Lagebericht ist explizit die Rede davon, die Datenlage erlaube keine fokussierten Maßnahmen in Bezug auf konkrete Personen (vgl. LKA NRW 2019, S. 8).
} 
Mit Wacquant gesprochen haben jedoch auch die „Spezialisten der kulturellen Produktion“" (Wacquant 2018, S. XXI) ihren Anteil an der Stigmatisierung des Stadtteils, darunter Journalist*innen der WAZ, die über Essen-Altendorf wiederholt skandalisierend berichteten. Die Zeitung behandelte entsprechende Vorfälle zunächst klassisch als „,crime as news“ (Hall et al. 1978, S. $66 \mathrm{ff}$.) und bediente dabei kulturalisierend-ethnisierende Stereotype. Berichterstattung und Artikelfrequenz folgt denselben Mustern, wie sie etwa Kearns et al. (2013) für Glasgow oder Wiest und Kirndörfer (2019) für Leipzig herausgearbeitet haben: Ihren „Peak“ erreicht die Berichterstattung immer dann, wenn spezifische Kriminalitätsereignisse dramatisiert werden können. Ergänzt wird die stadtteilbezogene Berichterstattung durch tendenziell wenige, anlassunabhängigere Zeitungsartikel, die auf positiv konnotierte Veränderungen und stadtpolitische Bemühungen verweisen oder „Multikulturalität“ preisen.

Es kann dabei begründet angenommen werden, dass die WAZ-Redaktion eine eigene Agenda skandalisierender Berichterstattung verfolgt. Denn auch wenn unserer methodologischen Perspektive entsprechend kein objektivierender Abgleich zwischen tatsächlichen Aktivitäten der Polizei, deren Pressemitteilungen und der lokalen Berichterstattung vorgenommen werden konnte, so ist die unterschiedliche Häufigkeit stadtteilbezogener öffentlicher Verlautbarungen von Polizei und medialer Thematisierungen von Kriminalität in Altendorf schwer zu verkennen. So übersteigt die Zahl der Berichte und Kommentare der WAZ zu Altendorf jene der polizeilichen Pressemitteilungen zu diesem Themenfeld um ein Vielfaches, insbesondere in Bezug auf Kriminalitätserzählungen: Von 135 öffentlich zugänglichen Pressemitteilungen der Polizei in den Jahren 2014-2019 (davon 42 ohne Kriminalitätsbezug) bezogen sich lediglich fünf auf Verstöße gegen das Betäubungsmittelgesetz. Der Begriff „Clan“ taucht lediglich einmal auf, nachdem 2019 innerhalb der nordrhein-westfälischen Polizei eine „Besondere Aufbauorganisation (BAO) Aktionsplan Clan“ geschaffen wurde. ${ }^{11}$ Für denselben Zeitraum lieferte die Online-Stichwortsuche bei der WAZ für die Kombination „Altendorf+Drogen“ 78 Treffer, für „Altendorf+Clan“ sogar 116 und die Kombination „Altendorf+Razzia“ förderte 71 Treffer zu Tage. ${ }^{12}$ Die WAZ setzt also deutliche eigene Schwerpunkte bei der Selektion ,berichtenswerter" Ereignisse. Daraus lässt sich gleichwohl nicht schlussfolgern, die Berichterstattung verliefe unabhängig von der oder gar gegen die Polizei: Polizeipräsident, Pressesprecher oder ortskundige Beamt*innen kamen als ,primary definers“ ebenso regelmäßig in den Artikeln zur Sprache wie die Innenminister von NordrheinWestfalen Ralf Jäger (SPD, bis 2017) und Herbert Reul (CDU, seit 2017).

Die in den Zeitungsartikeln dominierenden Schlagworte „Drogen“, „Clans“ oder auch „Razzia“ sind dabei ineinandergreifende „Verdichtungssymbole“ (Edelman 1976, S. 5), die breite Konnotationshöfe bereitstellen und starke emotionale Re-

\footnotetext{
11 Twitter-Meldungen der Polizei Essen sind überwiegend identisch mit den Mitteilungen über das Presseportal und werden deshalb hier vernachlässigt. Der erst 2014 eröffnete Account zeigt bis 2019 zudem zu Altendorf lediglich 19 Tweets.

12 Der Suchmodus begründet es, dass nicht zwangsläufig immer eine direkte Verbindung zwischen den gesuchten Begriffen besteht. Die absoluten Zahlen sind daher nur Orientierungswerte. Das Verhältnis von entsprechenden Pressemeldungen der Polizei und Zeitungsartikeln bleibt gleichwohl bestehen.
} 
aktionen hervorrufen. Über den Quartiers-, sprich Raumbezug werden sie weiter „,verdichtet“, sodass ein lokales Kontrollproblem erscheint (vgl. Mohring und Rolfes 2020, S. 119 sowie Hall et al. 1978, S. 329). ${ }^{13}$ Insbesondere das Schlagwort respektive die soziale Figur „Clan“ erlaubt es, höchst unterschiedliche Phänomene unter einem Label zu subsumieren und so aktuelle polizeiliche Schwerpunkteprogramme zu legitimieren. Gerade in seiner Offenheit ist der Begriff anschlussfähig an zahlreiche Assoziationsketten, die jeweils auf Gefährlichkeit verweisen. So rekurrieren die WAZ-Artikel auf ,ethnische“ Geschlossenheit und Loyalität, eine konstitutive Opposition zum Staat, Gewaltaffinität, eine große Mitgliederzahl von teilweise mehreren Tausend, auf archaische Lebensweisen und Wertvorstellungen, Paralleljustiz und vieles andere. ${ }^{14}$ Die kontrastierende Darstellung demonstriert dabei gleichzeitig ex negativo, was Deutschland, Essen und Altendorf „eigentlich“ ausmachen soll.

Anders als diese mediale Darstellungsweise suggeriert, positionieren sich die polizeilichen Akteur*innen jedoch ähnlich ambivalent zum negativen Image Altendorfs wie dies schon für die Stadtentwicklungspolitik gezeigt wurde. So wies der von uns interviewte Polizist auf deutlich abnehmende Kriminalität im Stadtteil hin und antwortete auf die Frage nach Problemen im Stadtteil: Da gebe es viele, zunächst aber müsse er kritisieren,

das Problem sind eigentlich die schnelllebigen Medien. [...] Weil jeder viel zu schnell Einfluss hat [...], so sind Sagen entstanden, weil jeder immer noch was dazu packt [...] umso krasser wird natürlich 'ne Situation und so kommt auch ein anderes Bild zustande (Exp_Adorf_05).

Der Polizeipräsident indes wird in einem WAZ-Artikel Anfang 2020 in Bezug auf vermeintlich zunehmende „Unsicherheitsgefühle“ im Stadtteil wie folgt zitiert: „Je mehr wir als Polizei machen, desto stärker wird wohl das Gefühl in der Bevölkerung, es passiere auch mehr“ (Maibaum 2020a). Insofern lässt sich eine negative Bedeutungsspirale beobachten, die auch die polizeiliche Arbeit an der "Sicherheit“ des Stadtteils immer wieder neu befördert. Diese Verbindung zwischen dem schlechten Ruf Altendorfs und den intensivierten Kontrollpraktiken zeigt sich nun auch auf unterschiedliche Weise in den Interviews mit Bewohner*innen, in denen Erzählungen erlebter Situationen wie auch Beschreibungen von Ereignissen im Stadtteil und allgemein bleibende Problematisierungen dazu dienen, das (nicht nur) territoriale Stigma zu managen.

\footnotetext{
${ }_{13}$ Clans werden vom Landeskriminalamt NRW wie in der medialen Darstellung auch über ihre vermeintliche „Beanspruchung regionaler oder krimineller Aktionsräume“ in „segregierten Räumen“ beschrieben (LKA NRW 2019, S. 6f.).

${ }^{14}$ Dieselben stereotypen und stigmatisierenden Beschreibungen finden sich auch in aktuell verwendeten Schulungsmaterialien der Polizei Essen (vgl. Polizeipräsidium Essen o.J.).
} 


\section{Stigmatisierung und Deutungen selektiver Kontrollen im Kontext der Stadtteilentwicklung}

Obwohl Altendorf medial sowie stadtpolitisch als Problemstadtteil und auch als gefährlich verhandelt wird, berichteten Interviewte auf unsere Frage nach persönlich erlebten Schwierigkeiten relativ selten über Konflikte mit anderen Bewohner*innen vor Ort. Vielmehr beschreiben sie häufig zuerst allgemeine Zuständen und beispielhafte Situationen, die als abweichend gedeutete Verhaltensweisen und ,Mentalitäten“ spezifischer Bewohner*innengruppen illustrieren sollten. Häufig tauchten dabei rassifizierende bzw. ethnisierende Gruppenkonstruktionen wie „die Libanesen“, „die Bulgaren und Rumänen“, „die Roma“ oder „die Afrikaner“ in Verknüpfung mit „Müll“, „Lärm“, „Drogen“ oder „Gewalt“ auf. Die entsprechenden Aussagen blieben jedoch ganz überwiegend allgemein und wurden nicht mit selbst erlebten „Kriminalitätsereignissen“ illustriert. Zudem kursierten Stadtteilgeschichten, die im kollektiven Gedächtnis präsent sind, und die teilweise mit Beschreibungen eigener Unsicherheit verknüpft werden: darunter prominent eine Messerstecherei, eine Schießerei, bei der ein bekannter Boxer verletzt wurde, und ein nachfolgender ,Angriff“ auf eine Pizzeria. Ergänzt wurden diese Geschichten mit Erzählungen über substrafrechtliche Devianzen wie abends lärmende Kinder, respektlose Jugendliche, „dicke Autos“ an zufahrtsbeschränkten Straßen oder liegengelassener Müll. Auf zugespitzte Weise scheinen sich hier die WAZ-Berichterstattung, allgemeine Problemdiskurse, wie sie seit den späten 2010er Jahren zirkulieren, und Erscheinungen zu vermischen, die in der Essener Stadtentwicklungspolitik problematisiert werden.

Abseits solcher, auch dramatisierender Schilderungen bemühen sich aber auch zahlreiche, sozial ganz unterschiedlich positionierte Bewohner*innen in den Interviews um eine „Normalisierung“ Altendorfs. Berichtet wird häufiger, dass hier jeder unter sich bleibe, aber auch von nachbarschaftlicher Solidarität und Engagement für den Stadtteil. Ein Bewohner Ende Zwanzig betont etwa, er sei ,hier wirklich noch nie auf der Straße dumm angemacht" worden, und auf die Erwähnung problematisierender Erzählungen anderer Bewohner*innen erklärt er diese als Eigenheit der „Älteren“: Er kenne das auch aus seiner Familie, das seien ,halt so die Generationen, die noch nicht in so einem vielfältigen Umfeld aufgewachsen sind, weil für uns ist das ganz normal: jugendliche Gruppen, am See, ganz gemischt, mit Migrationshintergrund und deutsche“"(Bew_Adorf_D_13).

Gleichzeitig finden sich vor allem in Erzählungen von Bewohner*innen of Color zahlreiche Hinweise auf alltägliche ,schwierige“ Begegnungen etwa mit Nachbar*innen, in denen rassistische Zuschreibungen eine deutliche Rolle spielen, ohne dass es zu wechselseitig konfrontativen Interaktionen - und insofern: zu Konfliktsituationen - käme (vgl. ausführlicher Rinn und Wiese 2020, für einen interaktionistisch orientierten Konfliktbegriff auch Mayer 2019). Überhaupt lässt sich festhalten, dass Bewohner*innen in Altendorf ihren Stadtteil als relativ konfliktarm erleben. ${ }^{15}$ Eine bedeutende Rolle spielte in den Antworten jedoch die wahrgenommene Polizei-

15 Etwas weniger als die Hälfte der Interviews enthalten keine Erzählungen über selbst erlebte schwierige Situationen und zehn Prozent der interviewten Bewohner*innen haben weder eigene Schwierigkeiten noch allgemeine Stadtteilprobleme zu berichten. 
präsenz, die diverse Interviewte von sich aus zur Sprache brachten: Polizeisirenen, Verkehrskontrollen oder Polizeieinsätze gegen „Kriminelle“ oder „Drogendealer“ tauchten regelmäßig in den Schilderungen auf. Umgekehrt berichteten andere interviewte Bewohner*innen von der eigenen Betroffenheit von Polizeikontrollen oder problematisierten diese zumindest.

$\mathrm{Ob}$ die Bewohner*innen Zeitungen wie die WAZ überhaupt lesen und wie sie entsprechende Berichte deuten, haben wir nicht systematisch erhoben. In den Interviews und ethnographischen Gesprächen wird deutlich, dass das Image Altendorfs als ,gefährliches Problemviertel“" den meisten Bewohner*innen zwar sehr wohl bewusst ist, jedoch unterschiedliche Lesarten der dortigen polizeilichen Aktivitäten zirkulieren. Neben zahlreichen affirmativen Aussagen trafen wir in einer geringeren Anzahl auf Bewohner*innen, die die Verbreitung des ,schlechten Rufs“ Altendorfs explizit kritisierten oder auch Gegenerzählungen vorbrachten. Die Ambivalenz und Breite der Rezeption polizeilicher Aktivitäten in Altendorf zeigt sich nicht nur zwischen unterschiedlichen Bewohner*innen, sondern oft sogar in ein und demselben Interview, wie etwa in einem mit zwei Jugendlichen (Bew_Adorf_D_20), die selektive Polizeipraktiken zunächst normalisieren und sodann Ausweiskontrollen detailliert beschreiben und als „Racial Profiling“ interpretieren:

Person A: Die kontrollieren hier die Jugendlichen, die sagen, wegen Betäubungsmitteln, Falschgeld, Waffen und so.

Person B: Und zum Beispiel, wenn du so eine Bauchtasche hast, so eine GucciTasche.

Interviewer: Echt, wieso das denn?

Person A: Weil die denken, du machst was mit Drogen. Neulich bin ich hier so langgegangen, habe in meine Bauchtasche reingefasst, wollte was rausholen, und dann hat die Polizei mich angehalten und wollten meinen Ausweis sehen. Ich so: „Warum kontrolliert ihr mich?“ Und die: „Weil in Altendorf so viel mit Betäubungsmittel gehandelt wird, und Falschgeld" und so weiter.

Interviewer: Und wie war das, also du hast da auch was zu den Polizisten gesagt?

Person A: Ja, das erste Mal, wo mir das passiert ist, da habe ich das gefragt. Aber ehrlich, also die Deutschen werden nie kontrolliert, also ich habe nichts gegen die Deutschen, aber es werden immer nur die Ausländer kontrolliert. (Bew_Adorf_D_20,179-190)

An verschiedenen Stellen des Interviews thematisierten die Jugendlichen das Zero-Tolerance-Policing, das sich Innenpolitik und Polizei NRW auf die Fahnen schreiben. Als Erscheinungen, die zu Kontrollen und Sanktionen führen, dominieren dabei „Müll“ und andere Disorder-Phänomene, die auch in der Stadtentwicklungspolitik problematisiert werden:

Interviewer: Und was passiert da so am See [Stadtentwicklungsprojekt Niederfeldsee, d. A.] unten?

Person A: Da gibt es auch Kontrollen von der Polizei. Aber vor allem vom Ordnungsamt. Jetzt in der letzten Zeit, das ist genauso wie da am Ehrenzeller Platz [weiteres Stadtentwicklungsprojekt, d. A.], da war es früher richtig dre- 
ckig, und jetzt ist es so, wenn du Pipa [geröstete Sonnenblumenkerne, d. A.] im Mund hast und dann wegwirfst, kommt das Ordnungsamt und du muss 30 bis 60 Euro zahlen. (Bew_Adorf_D_20, 258-262)

Zugleich bemerken sie jedoch auch:

Person A: Die Polizei hat Angst vor den Libanesen, deshalb machen die nichts, Angst vor den libanesischen Großfamilien. (Bew_Adorf_D_20,77-78)

Polizeiliche Aktivitäten werden mithin einerseits als Diskriminierung erlebt. Andererseits wird polizeiliche Inaktivität über die Zuschreibung von „Angst“ rationalisiert und der Clan-Diskurs aufgegriffen. Zugleich verweisen die Interviewten auf zwei zentrale Projekte der Quartiersentwicklung als Orte der Kontrollen - den umgestalteten Ehrenzeller Platz und den neu angelegten Niederfeldsee mit seiner hochpreisigen Neubebauung am Ufer. Gegenden im Umfeld dieser Aufwertungsprojekte werden mit verschärfter Kontroll- und Ordnungspolitik in Verbindung gebracht.

Auch drei weitere männliche Bewohner zwischen 20 und 40 Jahren äußern in einem Interview ihre ambivalente Sicht auf die Polizei und positionieren sich in Abgrenzung zu anderen Bewohner*innen auf der Seite der Anständigen (und dabei implizit als „deutsch“). So erzählen sie davon, selbst grundlos kontrolliert, mit zur Wache genommen und rabiat behandelt worden zu sein, als sie am Niederfeldsee saßen. Zugleich äußern sie Beschwerden, die Polizei käme spät oder ,mache nichts“, wenn man sie selbst riefe, wie etwa bei einer erlebten konflikthaften Situation mit Personen, die sie anti-romaistisch kategorisieren und von denen sie bedroht worden seien. Auch mangelndes polizeiliches Durchgreifen gegen „Afrikaner“ und Drogenhandel wird problematisiert (Bew_Adorf_D_02). Die eigene Betroffenheit von stadtteilbezogenen Kontrollpraktiken bestätigt so paradoxerweise rassistische Diskursstränge über Stadtteilprobleme und dafür verantwortlich gemachte Gruppen. Thematisierungen polizeilicher (In-)Aktivitäten werden somit in den Interviews für rassistische Differenzmarkierungen und eigene Identitätsdarstellungen genutzt.

Polizeiliche Interventionen und die damit assoziierte Entwicklung des Stadtteils werden in anderen Interviews prinzipiell begrüßt. So bemerkt etwa eine ca. 50jährige Bewohnerin, die seit 30 Jahren im Stadtteil wohnt, dass es ,,ruhiger geworden“ sei. Dass die Polizei viele Razzien durchführt, hält sie für begrüßenswert (Bew_Adorf_B_08, 25-29). Diese polizeilichen Aktivitäten deutet sie als Bestandteil der aufwertungsorientierten Stadtentwicklungspolitik:

Person C: Die machen das wegen Essen 51 [ein auf einer Brachfläche am Rand von Altendorf neu entstehender Stadtteil; d. A.], die räumen auf, die wollen hier ein anderes Publikum. Es muss sich auch verändern, damit die anderen Leute sich hier sicher fühlen. Das wird hier in den nächsten zwei, drei Jahren alles anders. (Bew_Adorf_B_08,30-33)

Ähnliche Deutungen zeigen sich in den Aussagen eines Bewohners Anfang Zwanzig. Auch er interpretiert die zunehmenden Polizeikontrollen explizit im Zusammenhang mit eben jenem Stadtentwicklungsprojekt „Essen 51“ und deutet sie als Teil einer intentionalen Verdrängungspolitik, die insbesondere „Schwarzköpfe“ wie ihn betreffe, die besonders häufig ,einfach so“, d.h. anlasslos kontrolliert würden: 
Person D: In Altendorf ist eigentlich nur der Ruf schlecht, also Altendorf hat einen schlechten Ruf, ist aber nicht so schlecht wie dieser Ruf, das wird alles in der Zeitung und im Internet verbreitet, das wird aufgebauscht, um Altendorf runterzuziehen, dass das hier alles kriminell ist, aber hier ist alles in Ordnung, hier passiert dir nichts, [...] das einzige was hier besonders ist in Altendorf ist die Polizeipräsenz, und die bauen da hinten ja auch einen neuen Stadtteil, wie heißt der?

Interviewer: Da hinten die Helenenstraße runter? Sie meinen bestimmt Essen 51.

Person D: Ja Essen 61 oder 51 oder wie das heißt. Ist doch klar, was die wollen, die wollen uns hier alle weghaben, rausschmeißen irgendwann, die sind ja nicht dumm, erst machen sie Altendorf runter, machen Altendorf einen schlechten Ruf, dann schicken sie viel Polizei rein, Polizeipräsenz, und nachher machen sie das hier alles platt, die wollen das hier ja alles vom Krupp-Gelände da aus neu machen hier. (Erhebungsprotokoll Anbahnung narrativ-episodale Interviews Altendorf, 18.4.2019_D)

Ein weiterer Bewohner, ebenfalls Anfang Zwanzig, den der Interviewer denjenigen zuordnet, die potenziell am ehesten polizeilich kontrolliert werden, schaltet sich während eines Interviewanbahnungsgesprächs spontan ein und weist dabei das von ihm antizipierte Thema des Interviews scharf zurück:

Person E: „Sie können hier alle fragen, fragen sie die Leute hier, es ist alles ganz normal hier, hier ist nicht mehr Kriminalität als in anderen Stadtteilen auch. ( $E r$ deutet rüber zur Kreuzung Helenenstraße/Altendorfer Straße): Sehen Sie hier Kriminalität? Schauen sie sich um, sehen sie etwas?“"

Interviewer: „Nee. Aber ich bin auch gar nicht wegen Kriminalität oder sowas hier, mich interessieren die persönlichen Erfahrungen der Menschen, hier in Altendorf zu wohnen, also was sie hier so täglich erleben, wie das Zusammenleben ist, und sowas.“

Person E: „Was ist denn an Altendorf so besonders? Warum gehen Sie nicht in jeden anderen Stadtteil, immer Altendorf, was ist denn das Besondere an Altendorf, hier ist alles ganz normal, ganz normal, außer hier die Polizei, die macht hier Stress. Was soll das, warum gehen Sie nicht woanders hin? Warum machen Sie das nicht da, wo Sie wohnen? [...]. Immer Altendorf. Hier gibt's keine Familien-Clans! In der Innenstadt, da sitzen die, da haben die ihre Häuser, in der Innenstadt, aber nicht hier in Altendorf, gehen sie doch da hin!“ (Erhebungsprotokoll Anbahnung narrativ-episodale Interviews Altendorf, 18.4.2019_D)

Diese rigorose Ablehnung der Beforschung Altendorfs deutet darauf hin, dass der Bewohner sich geradezu genötigt fühlt, seinen Stadtteil gegen den dominierenden Problemvierteldiskurs zu verteidigen. Naheliegenderweise nimmt er an, die Stigmatisierungen und insbesondere der Clan-Diskurs hätten uns nicht nur nach Altendorf geführt, sondern würden durch uns sozialwissenschaftlich oder journalistisch reproduziert. Kontrastierend zum hegemonialen Image sieht er das Problem nicht in der Kriminalität, sondern in der Polizei. Ohne diese wäre Altendorf ein ganz normaler Stadtteil. Aber, und das macht die Dringlichkeit seiner Zurückweisung deutlich, auch 
der einseitige problematisierende Fokus, der selektive öffentliche Blick auf Altendorf ist eine Zumutung und belastend. Und so zeigen sich in dieser Erhebungssituation auch die grundlegenden Ambivalenzen von Sozialforschung unter Bedingungen territorialer Stigmatisierung, deren Reproduktion, trotz möglicherweise gegensätzlicher forschungspolitischer Anliegen, nicht ausgeschlossen werden kann.

Entsprechend deutliche Zurückweisungen des medialen Problemimages Altendorfs zeigten sich auch in anderen Forschungsinteraktionen. Ein weiterer männlicher Interviewpartner beschreibt zudem Eskalationsdynamiken, die er unter anderem anhand der repressiven Polizeipraktiken während eines größeren Einsatzes verdeutlicht:

Person F: [erzählt, er sei kein „Bullenfreund“: Was er von denen schon alles für Gewalt erlebt habe, wenn man mal betrunken sei und jemanden beleidigt.] Dann ziehen die einem sofort einen über, und anzeigen kann man sie auch nicht, da hat man sofort Widerstand [...]. Und heute benutzen die ja auch sofort ihre Schusswaffe, hat einer ein Messer, ziehen die sofort ihre Pistole, ich meine, die haben Knüppel, die haben Gas, da müssen sie doch nicht gleich die Pistole ziehen. Aber hier in Altendorf, da trauen die sich nicht, [...] die kommen dann wenn nur noch vermummt, ganz in Schwarz, mit Maschinenpistolen und so. (Bew_Adorf_D_10,163-174)

Die hier aufgerufene typisierte eskalative Konstellation wurde etwa ein Dreivierteljahr nach diesem Interview gewissermaßen Realität: Am 18. Juni 2019 erschoss ein Polizist den Altendorfer Bewohner Adel B. im Eingangsbereich seines Wohnhauses (vgl. Rinn et al. 2020). Dieser hatte zuvor wegen suizidaler Absichten selbst die Polizei gerufen und trug ein Messer bei sich. Die Berichterstattung und die polizeiliche Positionierung zum Geschehen bestätigte nun einerseits das Image des „gefährlichen Problemviertels“ - „Polizist erschießt Messer-Mann in Essen“ schrieb die BILD-Zeitung (Weuster 2019). Andererseits provozierte der Fall verstärkt ab 2020 eine öffentliche Kontroverse zu institutionellem Rassismus und Polizeigewalt in Essen und Altendorf.

Auch eine 22-jährige in Altendorf aufgewachsene Bewohnerin betont in einem der narrativen Interviews das repressive, aus ihrer Perspektive überzogene polizeiliche Vorgehen im Stadtteil und verdeutlicht dies an einer erlebten Situation:

Person G: Mein Sohn kommt hochgerannt, der sagt: „Mama, die Polizei ist da auf dem Spielplatz“ [...]. Sie müssen sich jetzt vorstellen: Da stehen zwei Transporter, da stehen zwei Transporter, da steht ein Transporter, da stehen drei normale Wagen, zwei Motorräder. Sie müssen sich jetzt vorstellen, also hier waren bestimmt locker hundert bis zweihundert Polizisten. Wegen drei Jugendlichen. Die haben die Waffe gezogen. Haben die festgenommen. Weil am Essen-West [Bahnhof, d. A.] wurde da irgendwie ein Security-Mann angegriffen von drei Jugendlichen. Aber es waren die ja nicht. Allein, wie die damit umgegangen sind. Da denk ich mir so: Für drei Jugendliche brauch man doch keine zweihundert Polizisten [...]. (Na_Adorf_05_D, 51-58)

In der Suche nach einer Anschlussfähigkeit an Alltagserfahrungen lassen sich also Aneignungen und Reartikulationen dominanter Diskurse über Altendorf und 
damit verbundene polizeiliche Aktivitäten unterscheiden. Die verschiedenen Lesarten korrespondieren mit dem sozialen Status der Interviewten, mit ihren Klassenpositionen bzw. -erfahrungen, erlebtem Rassismus und Erfahrungen mit der Polizei (auch wenn sie sich nicht auf diese reduzieren lassen). Dabei bieten die Lesarten unterschiedliche „Gebrauchswerte“: Während die hegemoniale Erzählung des ,gefährlichen Problemviertels“ von vielen Interviewten überwiegend kritiklos und ohne Rekurs auf eigene Erfahrungen mit Konflikten mit anderen Stadtteilbewohner*innen aufgegriffen wird, bieten das territoriale Stigma und vor allem die Polizeipraktiken sowohl Chancen zur Distinktion gegenüber anderen Personengruppen als auch zur Kritik an der Polizei. So dient die Thematisierung der Stadtentwicklungspolitik wie gesehen im einen Fall der Legitimation, im anderen der Kritik an polizeilichen Aktivitäten.

Zusammenfassend lassen sich drei Varianten des Stigmamanagements unterscheiden. In der ersten Variante wird die Problematisierung Altendorfs insgesamt reproduziert, inklusive innerer Differenzierungen, indem das territoriale Stigma und Probleme auf spezifische konstruierte Gruppen zurückgeführt und somit Distinktionsgewinne erzielt werden. Erzählungen über eigene Erfahrungen mit Konflikten im Stadtteil, die dieses Bild untermauern würden, fehlen in dieser Variante allerdings weitgehend. Mitunter zeigt sich dieser Widerspruch zugespitzt in der Aussage, in Altendorf werde es immer schlimmer, aber selbst habe man hier noch nie etwas Schlimmes erlebt. Daneben zeigen sich zwei Varianten, die als Gegenerzählungen zum hegemonialen Diskurs begriffen werden können: Einerseits wird Altendorf als „ganz normaler“ und unproblematischer Stadtteil beschrieben. Statt Distinktion als Stigmaabwehr wird der Stadtteil an sich positiv dargestellt. Andererseits wird eine Gegenerzählung sichtbar, in der Altendorf nicht entproblematisiert wird. Statt in „Kriminalität“ wird das Problem und damit auch die Ursache der territorialen Stigmatisierung jedoch in der Polizei und im Rassismus gesehen und dies teilweise mit ausführlichen Schilderungen polizeilicher Aktivitäten illustriert.

Wacquants Diagnose der ,,verstellten“ Repräsentation scheint im Angesicht dieser Ergebnisse durchaus plausibel. Sie wirkt aber offenbar nicht ubiquitär, denn es werden Gegendiskurse sichtbar, in denen andere Repräsentationen sozialer Beziehungen im Stadtteil aufscheinen als jene, die den Problemviertel-Diskurs dominieren. Zugleich hat medial, politisch-administrativ und polizeilich prozessierte territoriale Stigmatisierung, so unsere Vermutung, deutliche Effekte auf den Raum des Denkund Sagbaren. Auch wenn wir letztlich keine Aussagen über die unmittelbaren Wirkungen des medialen Diskurses der WAZ und der über ihre Selektivität spezifische Be-deutungen nahelegenden Polizeipraktiken auf die Situationsdeutungen von Bewohner*innen machen können, so wurde in den Interviews doch eines deutlich: Der „schlechte Ruf“ und dessen Elemente, also die mit Altendorf verknüpften negativen Attribute, Phänomene, Personengruppen und Verhaltensweisen, werden von den Interviewpartner*innen gekannt. Korrespondenzen zwischen dem encoding und decoding der medialen Berichterstattung liegen hier somit ebenso nahe wie sich Interviewaussagen in den hegemonialen Stadtteildiskurs und entsprechend begründete stadtentwicklungs- und kriminalpolitische Programmatiken fügen respektive darauf (indirekt) beziehen. Allerdings determiniert der ,schlechte Ruf“, wie im Folgenden 
gezeigt wird, weder die Positionierung dazu noch die Interpretationen polizeilicher Aktivitäten.

\section{Situationsbe-deutungen und Wechselwirkungen}

Wie nun die diskursiv-praktische territoriale Stigmatisierung Altendorfs in konkreten Interaktionssituationen wirksam und reproduziert wird und sich durch diese hindurch Deutungshierarchien herstellen, kann ergänzend anhand zweier Ereignisse, die wir vor und während unserer Feldforschungen eher zufällig beobachteten, gezeigt werden. Dabei handelt es sich um eine in Begleitung eines Fernsehteams durchgeführte Razzia und den bereits erwähnten polizeilichen Großeinsatz. Neben den zitierten Interviewaussagen mag die Beobachtung der Razzia den Charakter nicht-affirmativer Deutungen von Polizeieinsätzen verdeutlichen. Dass Situationsbe-deutungen und daran anschließende Polizeiaktionen ,kontrafaktisch“ das stigmatisierende Image des Stadtteils bestätigen können, lässt sich wiederum abschließend anhand des eingangs zitierten WAZ-Artikels zeigen.

In der Pressemitteilung der Polizei Essen zur Razzia vom 15. Juni 2018 wird „Null Toleranz für Straftäter“ verkündet. Dort heißt es:

Seit April dieses Jahres finden verstärkt in der Essener Innenstadt und in Altendorf Kontrollmaßnahmen mit dem Ordnungsamt der Stadt Essen, dem Zollfahndungsamt und der Finanzverwaltung statt. [...] Mit einem Großaufgebot führten die Einsatzkräfte am Freitag (15. Juni) ab 17 Uhr bis nach Mitternacht Kontrollen in einschlägigen Bars, Geschäften und an bekannten szenetypischen Aufenthaltsorten durch. Insgesamt wurden 140 Personen, zahlreiche Fahrzeuge und 20 Objekte kontrolliert. Die Polizei vollstreckte drei Haftbefehle. 508 Verwarngelder wurden erhoben, 37 Ordnungswidrigkeiten und 10 Strafanzeigen wurden gefertigt. [...] Nur durch stetige Kontrollen können Straftaten reduziert und das Sicherheitsgefühl der Bürger gestärkt werden. (Polizei NRW Essen 2018)

In der WAZ erschienen in den folgenden Tagen drei Artikel, in denen es heißt, der Fokus habe diesmal auf Altendorf gelegen, ,wo sich seit Jahren Clans breitgemacht haben“. Der Pressesprecher der Polizei wird zitiert:

Wir tun alles, um keine Parallelgesellschaften aufkommen zu lassen und die Gesetze des deutschen Staates mit allen erlaubten Mitteln durchzusetzen. (Sat 2018)

Unseren Beobachtungen zufolge gingen einige Betroffene jedoch auf eine Weise mit der Kontrollsituation um, die die Situationsdeutung der WAZ-Redaktion geradezu konterkariert. In der Berichterstattung blieben diese Reaktionen entsprechend unsichtbar. Unser Beobachtungsprotokoll zeigt, wie sich die Betroffenen die Situation zumindest kurzfristig widerständig aneignen konnten:

Ein Polizist läuft von einem der Polizeibusse auf die Gruppe am Eingang [einer Bar in Altendorf, d. A.] zu, anschließend werden aus der Gruppe der Kon- 
trollakteure [...] Namen vorgelesen [...]. Ich deute die Gesamtsituation als Razzia, weil auch das Ordnungsamt und der Zoll dabei waren, es also etwa um Konzessionen, Hygiene und anderes gehen könnte (wie bei den Razzien, über die ich in der Presse gelesen hatte). Einer von den umstehenden Anwohnern auf der anderen Straßenseite spricht die beiden Presseleute [ein Kamerateam, d. A.] an [...]. Mir scheint, dass der Anwohner die Situation nicht wirklich ernst nimmt bzw. sich lustig macht. Die Gruppe junger Männer, die am Zaun neben dem Eingang lehnt, wirkt auf mich eher ruhig und gefasst, niemand schreit oder äußert lauten Unmut (was ich, da mir das aufgefallen ist, wohl erwartet habe). Einer von ihnen spricht allerdings mehrfach die Journalisten an. Erst scheint er mir zu „witzeln“, dann aber wird er ernster und sagt so etwas wie: „Es reicht, hört mal auf, ist gut jetzt““. Ein anderer ,Das wird nicht verpixelt? Dann kann jeder mein Gesicht im Internet sehen?“ Aus der Bar kommt ein Polizist [...] ein Einsatzleiter, so meine ich [...]. Die Razzia ist wohl beendet. Ganz zum Schluss läuft ein hochrangiger Polizist [...]. Er spricht mit dem Medientypen mit Mikro, sie scheinen sich zu kennen. Ich möchte ihn ansprechen, zögere erst und warte auf den richtigen Moment. [...] ,Entschuldigen Sie, ich wollte mal fragen, was hier passiert ist, gab es einen Vorfall?“. Er antwortet: „Nein, Routinekontrolle. Wir kontrollieren die Ausweise der Gäste, die Konzession, hier wird ja Tabak geraucht, wir prüfen, ob der auch versteuert ist“. „Also gab es jetzt keinen konkreten Vorfall“. „Nein, sowas machen wir hier regelmäßig (oder: gerade häufig?), es geht darum, hier für Recht und Ordnung zu sorgen. [...] Also hier gibt es ethnische Minder-, Gruppen, wir haben hier Probleme“" oder so etwas, er kann den Satz nicht zu Ende bringen, da hinter ihm sich eine Szene zwischen dem Kamerateam und einigen der jugendlichen Gäste abspielt, die ihn ablenkt. Einer der jungen Typen sagt: „Ey, mach nochmal die Kamera an“, das Kamerateam reagiert entsprechend, und der Typ beginnt zu ,,performen“: (X= er nennt einen Künstlernamen, glaube ich, ich verstehe den aber nicht richtig), ,mein Name auf Instagram; [X], mein Name auf Facebook“; ein anderer Gast zieht ihn aber von der Kamera weg und sagt, „Nee wir geben keine Interviews mehr“. [...] [Auf dem Weg wieder Richtung Altendorfer Str., d. A.] Drei jüngere Männer laufen vor [mir] und unterhalten sich laut, einer sagt: „Und das sollen Menschenrechte sein“, ein anderer: „Sogar meine Oma kann mich im Fernsehen sehn“; Auf der Straße stehen mehrere Autos, die noch von den parkenden Polizeiwagen blockiert werden; einer hupt, worauf einer der vor [mir] laufenden jungen Männer ruft: „Das ist nur die Polizei da vorne, hup nur, hup ordentlich“. An der Altendorfer Straße laufen wir in Richtung Haltestelle Röntgenstraße, und sehen auf der anderen Straßenseite weitere Polizeiwagen stehen, die offenbar eine weitere Razzia durchführen. (Feldnotizen Altendorf, 15.6.2018_D)

Verschiedene Betroffene interpretieren die Razzia und die gleichzeitige Präsenz eines offenbar im Vorfeld informierten Fernsehteams also durchaus unterschiedlich. Niemand jedoch begreift beides als gerechtfertigt oder vermeintlich notwendig. Deutungen als Verletzung der Privatsphäre und als Menschenrechtsverletzung stehen neben Beschwerden über Störungen und Straßenblockaden, die zu protestierendem 
Hupen herausfordern. Wie in den oben zitierten Beispielen der Kritik von Bewohner*innen an überzogenen, selektiven und diskriminierenden Polizeipraktiken, die wie gesehen teils direkt mit der Aufwertungspolitik verknüpft werden, zeigen sich auch hier mithin „Counter-narratives“ (Garbin und Millington 2012, S. 2074). Diese werden jedoch nicht wirkmächtig über die einzelnen Äußerungen hinaus: Selbst die Aneignung der Situation zur Selbstinszenierung taucht in der medialen Öffentlichkeit nicht auf.

Da die Deutungen außerhalb individueller Posts auf Social-Media-Plattformen über die verortete Situation hinaus nicht sichtbar werden, können sie keine Wirkung auf das hegemoniale Stadtteilimage entfalten. Die Kontrollierenden behalten somit die Definitionsmacht hinsichtlich der Interpretation des Geschehenen. Die Erfahrungen mit der Razzia - oder auch andere Erfahrungen mit der Polizei - stellen für die Kontrollierten jedoch Deutungsfolien bereit, mit denen sie wiederum zukünftige polizeiliche Aktivitäten interpretieren. Dies begründet die teilweise deutlichen Zurückweisungen der öffentlichen Darstellung Altendorfs und der Polizei, wie sie auch in Teilen der Interviews zum Ausdruck kamen.

Im Falle des eingangs erwähnten „Fehlalarms“ bildeten vermutlich die Erwähnung von Schüssen in der Meldung des Anrufers, das polizeiliche Wissen um vergangene Ereignisse im Stadtteil und eine Auseinandersetzung in einem in einem anderen Essener Stadtteil gelegenen Café einige Tage zuvor erste Kontextbedingungen für die Entscheidung der lokalen Polizeileitung, mit einem Großaufgebot (,massive Kräfte“, Kintscher 2017) vor Ort an der „,berüchtigten“ Altendorfer Straße, die das Stadtteilzentrum bildet, zu erscheinen. Während der Beobachtung des abendlichen Einsatzes zeigten sich verschiedene affirmative, aber auch differenzierende Deutungen der Polizeipräsenz. Eine Passantin kommentierte den Einsatz mit „es wird immer schlimmer hier“, wobei sie nicht den Polizeieinsatz, sondern den vermuteten Anlass zu meinen schien. In einer alteingesessenen Pizzeria beruhigte der Inhaber eine Kundin dahingehend, dass „das“ nur etwas „unter denen“ sei. Obgleich auch hier scheinbar selbstverständliche Gruppenkonstruktionen aufgerufen wurden, diente die Abgrenzung dabei weniger der Degradierung von „denen“, als vielmehr der Beruhigung ob der dramatisch wirkenden Polizeipräsenz. Aufgrund der Präsenz von mehreren, mit migrantisch gelesenen Männern besetzten SUVs spekulierte ebenso der beobachtende Sozialforscher, diese wären wegen des Polizeieinsatzes oder dessen Anlass gekommen.

Vom Anrufer, der die vermeintlichen Schüsse und die sich „zusammenrottenden“ Männer meldete, über die Polizei, die Passantin, den Imbissbetreiber, mutmaßlich die SUV-Besatzungen und den Sozialforscher erfolgten die Interpretationen der Situationen auf Basis von Annahmen, die die Anwesenden bereits mitbrachten - und „nicht aufgrund des Augenscheins“ (Edelman 1976, S. 103). Das zuvor diskursiv produzierte und zirkulierende Wissen leitete die Interpretationen. Es handelt sich also um ein klassisches Thomas-Theorem-Phänomen, das zirkuläre Effekte zeitigt: "If men define situations as real, they are real in their consequences“ (Thomas und Thomas 1928). Auf Basis ihres Wissens über den Stadtteil interpretierten die Beteiligten die Situationen und handelten daran anschließend die Situationsbe-deutungen interaktiv aus. Eine bedeutungsoffene Situation (laute Geräusche und herumstehende Personen) wurde so ,typisiert“", weil sie sich scheinbar in das Bild von Altendorf 
und daran anknüpfende (polizeiliche) Handlungsrepertoires fügte. Gesellschaftliche Autorität und Definitionsmacht der Institution Polizei legitimierten den Einsatz ohne Grund, so ließ sich unterstellen, wäre sie ja nicht gekommen. Zugleich reproduzierte dieses Vorgehen das Altendorf-Image als Interpretationsrahmen für zukünftige Ereignisse. Die Stellungnahme des Essener Polizeisprechers, demzufolge es darum gehe, „Flagge zu zeigen, deutlich zu machen, dass wir in der Stadt keine Paralleljustiz dulden“, hatte daran ebenso Anteil. ${ }^{16}$

Die Relevanz von Vorstellungen über erwartbare Ereignisse im Stadtteil - dies ist Kern dessen, was Stadtteilimages ausmachen - ist der Polizei dabei durchaus bewusst. Im bereits zitierten Expert*inneninterview sieht der Polizeibeamte sich jedoch selbst nicht als Teil dieser Wissensproduktion, sondern kritisiert neben der Medienberichterstattung die Anzeige- bzw. Meldebereitschaft der Wohnbevölkerung:

Einer hört mal nachts ein lauteres Auto und sofort „,die ganze Clan-Welt eiert wieder mit so nem Auto durch die Gegend“. Das ist natürlich grundsätzlich falsch [...]. (Exp_Adorf_05). ${ }^{17}$

Gleichwohl wird die territoriale Stigmatisierung der Bewohner*innen durch mediale Berichterstattung und polizeiliches Handeln reproduziert: Altendorf wird als gefährlicher, devianter, desintegrierter Raum markiert, der noch intensiver als ohnehin polizeilich überwacht werden muss. Insbesondere kulturalisierend oder ethnisierend und damit rassistisch markierte Stadtteilbewohner*innen und -nutzer*innen bekommen dies in ihren alltäglichen Aneignungspraktiken zu spüren. Nicht nur tatsächliche Gewaltereignisse können Nachbarschaften symbolisch in ,a specter of hostile racial ghetto ready to erupt in mayhem any minute" (Wacquant et al. 2014, S. 1274) verwandeln, sondern bereits polizeiliche Reaktionen auf entsprechende Erwartungen und Berichterstattungen. Die „symbolische Gewalt“ des territorialen Stigmas, auf die nicht nur Wacquant verweist, betrifft dabei alle Bewohner*innen Altendorfs - aber nicht alle gleich. Sie eröffnet oder begrenzt Möglichkeiten, sich dazu zu positionieren, auf sozial ungleiche Weise. Das zum einen, weil manche Positionierungen anschlussfähiger an hegemoniale Problemviertel-Diskurse sind, zum anderen, weil die Betroffenheit von Polizeikontrollen auch im „Gefahrengebiet“ ungleich verteilt ist. Insbesondere die polizeiliche Kernklientel der männlichen, mit Insignien einer ,Straßenkultur“ ausgestatteten und in den meisten Fällen migrantisierten ,üblichen Verdächtigen“ (vgl. auch Künkel 2014), bekommen territoriale Stigmatisierung in ihren alltäglichen Nutzungen des Stadtteils zu spüren.

\footnotetext{
16 Wie relevant sehr kurzfristige Situationsdefinitionen durch die Polizei für das weitere Geschehen sind, lässt sich am Beispiel der Schüsse in und bei einem Einkaufszentrum in München 2016 zeigen: Nicht nur die unmittelbar einsatztaktisch relevante Frage, ob es denn neonazistischer „Terrorismus“ oder ein „Amoklauf“ war, wurde noch Monate später diskutiert. Durch die Ad-Hoc-Berichterstattung und Posts in den sozialen Medien wurden je nach Rekonstruktion 67 bis 73 „Phantomtatorte“ geschaffen, auf die wiederum polizeilich reagiert wurde (vgl. Backes et al. 2016).

17 Vgl. auch die Meldung, bei der eine Hochzeitgesellschaft in Altendorf als Clankonflikt gedeutet wurde: WAZ 2019.
} 


\section{Fazit: Verstärkerkreisläufe und Deutungshoheiten}

In Bezug auf die Konstruktion des „Problemviertels“ Altendorf ergibt sich eine Triade der Bedeutung aus Medienberichten, politisch-polizeilichen Programmen und polizeilichen Praktiken vor Ort. Persönliche Erfahrungen werden vor dem Hintergrund und in Wechselwirkung mit diesen Elementen interpretiert. Interaktionistisch gedeutet wird der „gefährliche“ und ,problematische Stadtteil“ in direkt oder indirekt aufeinander bezogenen Handlungen hervorgebracht, die sich wechselseitig verstärken. Ein Ausgangspunkt ist dabei nicht rekonstruierbar. Unabhängig von Anlässen und Hintergründen kommt polizeilichen Handlungen und der polizeilichen Definitionsmacht eine symbolische Funktion für die Reproduktion des territorialen Stigmas und der Rahmung Altendorfs als „Problemviertel“ zu: durch ihre Kontrollpraktiken und die Definition von Situationen als Ordnungswidrigkeit oder gar ClanKriminalität sowie bei der späteren medialen Repräsentation von Ereignissen und Situationen als potenziell gefährlich für die stadtgesellschaftliche Kohäsion. Wie gesehen müssen auch kriminal- und ordnungspolitische Interventionen zur Kriminalitätsbekämpfung und zur Aufwertung des Stadtteils in diesem Sinne verstanden werden. Sie reproduzieren dabei nicht nur das Stadtteilimage, sondern konterkarieren paradoxerweise gleichzeitig die Programmatik der Stadtteilentwicklung: Wo offensiv poliziert wird, wird das umworbene „bürgerliche“ Klientel skeptisch. Auch den stadtpolitischen Initiativen müssen stigmatisierende und ausschließende Effekte zugeschrieben werden, besonders hinsichtlich der von urbaner Marginalisierung am stärksten betroffenen Bewohner*innen.

Für Essen-Altendorf bietet sich damit abschließend die Deutung der genannten Prozesse als zirkulär an. Seit über 20 Jahren dominiert eine Verfallserzählung über den Stadtteil, die durch medial vermittelte Skandalisierung von Kriminalität verstärkt wird. Vermeintliche Evidenz erhält dies durch Einzelereignisse im Stadtteil, die selbst erlebt werden oder über die berichtet wird. Die medial seit Jahrzehnten wiederholte Assoziation von als migrantisch markierten Menschen mit Kriminalität beziehungsweise mit spezifischen Konfliktkonstellationen wird auch in Altendorf "located and situated" (Hall et al. 1978, S. 329, Hervorh. i. Orig.). Über Prozesse „der interpretierenden Selbstinteraktion“ (Mayer 2019, S. 53) erfolgt die Deutung von „Dingen“, d.h. von Situationen und ihren konstitutiven Elementen, im Register des territorialen Problemdiskurses: Die jeweils vorangegangenen Bedeutungen rahmen wiederum neue Situationsdefinitionen und die daran anschließenden Interpretationen und Handlungen. Politische Programmatiken polizeilichen, aber auch sozialarbeiterischen Handelns, sowie dominante Formen der Berichterstattung befördern eine tendenzielle Institutionalisierung von Bedeutungen (vgl. auch Christmann 2013, S. 159).

Polizeiliche Interventionen und Bearbeitungen von Alltagskonflikten werden institutionalisiert, da einerseits diskursiv mit gravierenden Handlungen wie Messerstechereien oder Schießereien gerechnet wird. Andererseits wird polizeilich im Anschluss an „Broken Windows"-Konzepte und die „Strategie der 1000-Nadelstiche“ bereits bei substrafrechtlichen Normverstößen „konsequent" interveniert. Zusammen mit der Berichterstattung entsteht frei nach Sebastian Scheerer (1978) somit ein polizeilich-publizistischer Verstärkerkreislauf, aus dem wiederum konkrete poli- 
zeiliche Praxen folgen. Rassistische Problematisierungsdiskurse und institutioneller Rassismus bestätigen sich so gegenseitig. Bedeutungen materialisieren sich in Handlungen: Jede einzelne Polizeipräsenz, jeder einzelne Zeitungsartikel wird zum Beleg für die „Richtigkeit der Abstraktion“ (Stehr 1998, S. 68). Während Deutungen als „Racial Profiling“, Schikane oder Polizeigewalt politisch-medial kaum gehört oder gar diskreditiert werden, sind affirmative Lesarten von Berichten und Ereignissen hegemonial. ${ }^{18}$

Angesichts einer solchen „Hierarchie der Glaubwürdigkeit“ (Becker 2016 [1967], S. 12) ist es auch eine der Aufgaben von Wissenschaft, nicht nur herrschaftliche Politiken und Praktiken als solche zu benennen, sondern eben auch andere Perspektiven „von unten“ und auch in ihrer Ausdifferenziertheit und ihren (Macht-)Effekten - hier auf Beziehungen unterschiedlicher Quartiersbewohner*innen zueinander - sichtbar zu machen: Nicht weil sie statt der herrschenden „wahrer“ oder ,sympathischer“ wären, sondern - so Heinz Steinert (1998) - weil eine Annäherung an Wahrheit nur über die Perspektivenvielfalt möglich ist und erst dadurch Ungleichheit und Herrschaftsverhältnisse in den Blick geraten. Das war auch die Idee der Frage von Howard S. Becker (2016 [1967]): „Whose side are we on?“

Förderung Gefördert durch die Deutsche Forschungsgemeinschaft (DFG), Projektnummer 389360901.

Funding Open Access funding enabled and organized by Projekt DEAL.

Open Access Dieser Artikel wird unter der Creative Commons Namensnennung 4.0 International Lizenz veröffentlicht, welche die Nutzung, Vervielfältigung, Bearbeitung, Verbreitung und Wiedergabe in jeglichem Medium und Format erlaubt, sofern Sie den/die ursprünglichen Autor(en) und die Quelle ordnungsgemäß nennen, einen Link zur Creative Commons Lizenz beifügen und angeben, ob Änderungen vorgenommen wurden.

Die in diesem Artikel enthaltenen Bilder und sonstiges Drittmaterial unterliegen ebenfalls der genannten Creative Commons Lizenz, sofern sich aus der Abbildungslegende nichts anderes ergibt. Sofern das betreffende Material nicht unter der genannten Creative Commons Lizenz steht und die betreffende Handlung nicht nach gesetzlichen Vorschriften erlaubt ist, ist für die oben aufgeführten Weiterverwendungen des Materials die Einwilligung des jeweiligen Rechteinhabers einzuholen.

Weitere Details zur Lizenz entnehmen Sie bitte der Lizenzinformation auf http://creativecommons.org/ licenses/by/4.0/deed.de.

\section{Einhaltung ethischer Richtlinien}

Interessenkonflikt M. Rinn und J. Wehrheim geben an, dass kein Interessenkonflikt besteht.

Ethische Standards Für diesen Beitrag wurden von den Autoren keine klinische Studien an Menschen oder Tieren durchgeführt. Für die aufgeführten Studien gelten die jeweils dort angegebenen ethischen Richtlinien.

\footnotetext{
18 Ein weiteres Beispiel dafür: Im Frühjahr 2020 kommunizierten Oberbürgermeister und Polizeipräsident via Pressemitteilungen eine Strafanzeige wegen Beleidigung der Polizei, weil auf einer Demonstration Vorwürfe rassistischer Polizeikontrollen laut wurden. Die WAZ stützte diese rigorose Reaktion sogleich in ihren Kommentaren (vgl. Maibaum 2020b).
} 


\section{Literatur}

\section{Verwendete Literatur}

Amjahid, M. (2020). So schnell wird man zum Clan-Kriminellen. Zeit.de vom 26. Mai 2020. URL: https://www.zeit.de/amp/gesellschaft/2020-05/diskriminierung-clan-kriminalitaet-razzien-polizeirassismus. Zugegriffen: Juni 2021.

Backes, T., Jaschensky, W., Langhans, K., Munzinger, H., Witzenberger, B., \& Wormer, V. (2016). Timeline der Panik. Ein Täter, ein Tatort - und eine Stadt in Angst: Wie aus dem Münchner Amoklauf ein Terroranschlag mit 67 Zielen wurde. Eine Rekonstruktion. Sueddeutsche.de vom 1. Okt. 2016. URL: https://gfx.sueddeutsche.de/apps/57eba578910a46f716ca829d/www. Zugegriffen: Juni 2021.

Bareis, E., \& Cremer-Schäfer, H. (2013). Empirische Alltagsforschung als Kritik. Grundlagen der Forschungsperspektive der „Wohlfahrtsproduktion von unten“. In G. Graßhoff (Hrsg.), Adressaten, Nutzer, Agency. Akteursbezogene Forschungsperspektiven in der Sozialen Arbeit (S. 139-159). Wiesbaden: Springer VS.

Baum, M., \& Otto, M. (2020). Die Paradoxie raumbezogener Sozialplanung. Zum Prozess nichtintendierter territorialer Stigmatisierung durch Sozialberichterstattung. Leviathan, 48, 237-263.

Becker, H. S. (2014) [1963]. Außenseiter. Zur Soziologie abweichenden Verhaltens. Wiesbaden: Springer VS.

Becker, H. S. (2016) [1967]. „Whose Side Are We On?“. In D. Klimke \& A. Legnaro (Hrsg.), Kriminologische Grundlagentexte (S. 7-22). Wiesbaden: Springer VS.

Belina, B. (2018). Wie Polizei Raum und Gesellschaft gestaltet. In D. Loick (Hrsg.), Kritik der Polizei. (S. 119-133). Frankfurt a. M.: Campus.

Belina, B., \& Wehrheim, J. (2011). „Gefahrengebiete“. Durch die Abstraktion vom Sozialen zur Reproduktion gesellschaftlicher Strukturen. Soziale Probleme, 23, 207-229.

Blumer, H. (1981). Der methodologische Standpunkt des symbolischen Interaktionismus. In Arbeitsgruppe Bielefelder Soziologen (Hrsg.), Alltagswissen, Interaktion und gesellschaftliche Wirklichkeit 1+2 (S. 80-146). 5. Auflage. Opladen: Westdeutscher Verlag.

Böhme, A. (2019). Polizei vermeldet erste Erfolge im Kampf gegen Clans. WAZ.de vom 10. Dez. 2019. URL: https://www.waz.de/staedte/essen/essen-polizei-formiert-siko-ruhr-fuer-kampf-gegen-clansid227873823.html. Zugegriffen: Juni 2021.

Boller, M. (2013). „Stadt im Kopf“ - Überlegungen aus einer mehrperspektivischen Betrachtung im Stadtteil Köln-Mülheim. In O. Schnur, P. Zakrezewski \& M. Drilling (Hrsg.), Migrationsort Quartier. Zwischen Segregation, Integration und Interkultur (S. 89-106). Wiesbaden: Springer VS.

Bourdieu, P. (1998a). Ortseffekte. In V. Kirschberg \& A. Göschel (Hrsg.), Kultur in der Stadt. Stadtsoziologische Analysen zur Kultur (S. 17-25). Opladen: Leske + Budrich.

Bourdieu, P. (1998b). Die rechte und die linke Hand des Staates. In P. Bourdieu (Hrsg.), Gegenfeuer (S. 12-21). Konstanz: UVK.

Bridge, G., Butler, T., \& Lees, L. (2012). Mixed communities. Gentrification by stealth? Bristol: Policy Press.

Bröckling, M. (2018). Influencer in Uniform: Wenn die Exekutive viral geht. Netzpolitik.org vom 5. März 2018. URL: https://netzpolitik.org/2018/wenn-die-exekutive-viral-geht-twitter-wird-zum-lieblingswerkzeug-der-deutschen-polizei/. Zugegriffen: Juni 2021.

Christmann, G. B. (2013). Raumpioniere in Stadtquartieren und die kommunikative (Re-)Konstruktion von Räumen. In R. Keller, H. Knoblauch \& J. Reichertz (Hrsg.), Kommunikativer Konstruktivismus. Theoretische und empirische Arbeiten zu einem neuen wissenssoziologischen Ansatz. (S. 153-184). Wiesbaden: Springer VS.

Dangschat, J. S. (1997). Sag' mir, wo Du wohnst, und ich sag' Dir, wer Du bist! Zum aktuellen Stand der deutschen Segregationsforschung. PROKLA. Zeitschrift für Kritische Sozialwissenschaft, 27(109), 619-647.

DHPol (Deutsche Hochschule der Polizei) (2020). Sicherheitsanalysen und -vernetzung für Stadtquartiere im Wandel (SiQua). Auswertungen zur Polizeilichen Kriminalstatistik für Stadtbezirke und Stadtteile in Essen. URL: http://www.si-qua.de/sites/default/files/2020-03/hellfeld-essen-2020-03-16.pdf. Zugegriffen: Juni 2021.

Edelman, M. (1976). Politik als Ritual. Die symbolische Funktion staatlicher Institutionen und politischen Handelns. Frankfurt a. M.: Campus.

Fassin, D. (2018). Die Politik des Ermessenspielraums: Der „graue Schreck“ und der Polizeistaat. In D. Loick (Hrsg.), Kritik der Polizei (S. 135-164). Frankfurt a. M.: Campus. 
Feest, J., \& Blankenburg, E. (1972). Die Definitionsmacht der Polizei. Strategien der Strafverfolgung und soziale Selektivität. Düsseldorf: Bertelsmann Universitätsverlag.

Garbin, D., \& Millington, G. (2012). Territorial stigma and the politics of resistance in a Parisian banlieue: La courneuve and beyond. Urban Studies, 49, 2067-2083.

Gestring, N. (2012). Images von Migrantenquartieren: Ausgrenzung durch Stigmatisierung? In F. Kraas \& T. Bork (Hrsg.), Urbanisierung und internationale Migration. Migrantenökonomien und Migrationspolitik in Städten (S. 97-110). Baden-Baden: Nomos.

Glaser, J. (2015). Suspect race. Causes and consequences of racial profiling. Oxford: Oxford University Press.

Glasze, G., Pütz, R., \& Rolfes, M. (2005). Verräumlichung von (Un-)Sicherheit, Kriminalität und Sicherheitspolitiken - Herausforderungen einer Kritischen Kriminalgeographie. In G. Glasze, R. Pütz \& M. Rolfes (Hrsg.), Diskurs - Stadt - Raum (S. 13-58). Bielefeld: transcript.

Goffman, E. (1967). Stigma. Über die Techniken der Bewältigung beschädigter Identität. Frankfurt a. M.: Suhrkamp.

Hagenbucher, R. (2017). Schulleiterin klagt: „Unsere Arbeit ist nicht zu schaffen“. WAZ.de vom 19. Jan. 2017. URL: https://www.waz.de/staedte/essen/borbeck-und-west/experten-werfen-unterschiedlicheblicke-auf-altendorf-id209333421.html. Zugegriffen: Juni 2021.

Hall, S., Critcher, C., Jefferson, T., Clarke, J., \& Roberts, B. (1978). Policing the crisis. Mugging, the state, and law and order. Basingstoke: Macmillan.

Hanak, G., Stehr, J., \& Steinert, H. (1989). Ärgernisse und Lebenskatastrophen. Über den alltäglichen Umgang mit Kriminalität. Bielefeld: AJZ.

Häußermann, H., \& Kronauer, M. (2009). Räumliche Segregation und innerstädtisches Ghetto. In R. Stichweh \& P. Windolf (Hrsg.), Inklusion und Exklusion: Analysen zur Sozialstruktur und sozialen Ungleichheit (S. 157-173). Wiesbaden: VS Verlag für Sozialwissenschaften.

Heitmeyer, W. (1998). Versagt die „Integrationsmaschine Stadt“? Zum Problem der ethnischkulturellen Segregation und ihrer Konfliktfolgen. In W. Heitmeyer, R. Dollase \& O. Backes (Hrsg.), Die Krise der Städte (S. 443-467). Frankfurt a. M.: Suhrkamp.

Hunold, D. (2015). Polizei im Revier. Polizeiliche Handlungspraxis gegenüber Jugendlichen in der multiethnischen Stadt. Berlin: Duncker \& Humblot.

Kearns, A., Kearns, O., \& Lawson, L. (2013). Notorious places: Image, reputation, stigma. The role of newspapers in area reputations for social housing estates. Housing Studies, 28, 579-598.

Keitzel, S. (2020). Varianzen der Verselbstständigung der Polizei per Gesetz. Kriminologisches Journal, 52, 191-209.

Kintscher, W. (2017). Sicherheit. Nach der Randale: Polizei Essen will gezielt Stärke zeigen. WAZ.de vom 8. Dez. 2017. URL: https://www.waz.de/staedte/essen/nach-der-randale-polizei-will-gezieltstaerke-zeigen-id212780377.html. Zugegriffen: Juni 2021.

Künkel, J. (2014). Cop Culture Reloaded? Wandel und Persistenzen schutzpolizeilicher Macht. Kriminologisches Journal, 46, 264-283.

Landtag Nordrhein-Westfalen (2020). Antwort der Landesregierung auf die Kleine Anfrage 3490 vom 20. April 2020 des Abgeordneten Sven W. Tritschler AfD, Drucksache 17/9022: Gefährliche Orte: Warum missachtet die Landesregierung das Urteil des Verfassungsgerichtshofs vom 28. Januar 2020? Drucksache 17/9401. URL: https://www.landtag.nrw.de/portal/WWW/dokumentenarchiv/ Dokument/MMD17-9401.pdf. Zugegriffen: Juni 2021.

Lindner, R. (2004). Walks on the wild side. Eine Geschichte der Stadtforschung. Frankfurt a. M.: Campus.

LKA NRW (Landeskriminalamt Nordrhein-Westfalen) (2019). Clankriminalität - Lagebild NRW 2018. URL: www.polizei.nrw. https://polizei.nrw/sites/default/files/2019-05/190515_Lagebild\%20Clan \%202018.pdf. Zugegriffen: Juni 2021.

Maibaum, J. (2019a). Essener Behörden gelingt neuer Schlag gegen Clankriminalität. WAZ.de. vom 10. Dez. 2019. URL: https://www.waz.de/staedte/essen/essener-behoerden-gelingt-neuer-schlag-gegenclankriminalitaet-id227879069.html. Zugegriffen: Juni 2021.

Maibaum, J. (2019b). Essen: Verhaftung bei Razzia gegen Clan-Kriminalität. WAZ.de vom 25. Dez. 2019. URL: $\quad$ https://www.waz.de/staedte/essen/essen-verhaftung-bei-razzia-gegen-clan-kriminalitaetid227913691.html. Zugegriffen: Juni 2021.

Maibaum, J. (2020a). Studie zeigt: Wo sich die Essener am unsichersten fühlen. WAZ.de vom 29. Jan. 2020. URL: https://www.waz.de/staedte/essen/studie-zeigt-wo-sich-die-essener-am-unsicherstenfuehlen-id228276059.html. Zugegriffen: Juni 2021.

Maibaum, J. (2020b). Der Vorwurf rassistischer Motive ist eine linke Groteske. WAZ.de vom 26. Feb. 2020. URL: https://www.waz.de/staedte/essen/der-vorwurf-rassistischer-motive-ist-eine-linke-groteskeid228555531.html. Zugegriffen: Juni 2021. 
Mayer, L. (2019). Konfliktdynamiken - Kriegsdynamiken. Zur Konstitution und Eskalation innergesellschaftlicher Konflikte. Bielefeld: transcript.

Mohring, K. \& Rolfes, M. (2020). No-go-Areas. Wie eine Raumsemantik zwischen Politik und Massenmedien vermittelt und Räume rechter Gewalt zu Räumen ethnisierter Kriminalität werden. Geographische Zeitschrift, 108, 99-124.

Morris, L. (1994). Dangerous classes. The underclass and social citizenship. London, New York: Routledge.

Münch, S. (2014). Das „Mantra der Mischung“: Die Problematisierung von ethnischer Segregation in Deutschland und den Niederlanden. In P. Gans (Hrsg.), Räumliche Auswirkungen der internationalen Migration (S. 327-343). Hannover: ARL.

Peters, H. (2009). Devianz und soziale Kontrolle. Weinheim: Juventa.

Polizei NRW Essen (2018). POL-E: Essen: Erneute Kontrollmaßnahmen in Altendorf und der nördlichen Innenstadt - „Null-Toleranz““ für Straftäter. URL: www.presseportal.de vom 17. Juni 2018. URL: https://www.presseportal.de/blaulicht/pm/11562/3972798. Zugegriffen: Juni 2021.

Polizeipräsidium Essen (o.J.). Arabische Familienclans. Historie. Analyse. Ansätze zur Bekämpfung. https://www.presseportal.de/download/document/707785-brosch-re-arabische-clans.pdf. Zugegriffen: Juni 2021.

Reinecke, C. (2021). Die Ungleichheit der Städte. Urbane Problemzonen im postkolonialen Frankreich und der Bundesrepublik. Göttingen: Vandenhoeck \& Ruprecht.

Rinn, M., \& Wiese, L. (2020). Politiken sozialer Mischung und die Produktivität von Rassismus im ,gefährlichen Viertel“. Geographica Helvetica, 75(1), 23-36.

Rinn, M., Wehrheim, J., \& Wiese, L. (2020). Kein Einzelfall. Über den Tod von Adel B., der während eines Polizeieinsatzes in Essen-Altendorf erschossen wurde. sub\urban - Zeitschrift für kritische Stadtforschung, 8(1/2), 263-276.

Ronneberger, K., \& Tsianos, V. (2009). Panische Räume. Das Ghetto und die „Parallelgesellschaft“. In S. Hess, J. Binder \& J. Moser (Hrsg.), No integration?! Kulturwissenschaftliche Beiträge zur Integrationsdebatte in Europa (S. 137-152). Bielefeld: transcript.

Ruch, A. (2017). Polizeiliche Entscheidungsspielräume als Einfallstor für Diskriminierung. Monatsschrift für Kriminologie und Strafrechtsreform, 100, 328-343.

Sack, F. (1968). Neue Perspektiven in der Kriminologie. In F. Sack \& R. König (Hrsg.), Kriminalsoziologie (S. 431-475). Frankfurt a. M.: Akademische Verlagsgesellschaft.

Sat, S. (2018). Wieder Razzia gegen Clans in Essen - Politik der Nadelstiche. WAZ.de vom 17. Juni 2018. URL: https://www.waz.de/staedte/essen/wieder-razzia-gegen-clans-in-essen-politik-dernadelstiche-id214610023.html. Zugegriffen: Juni 2021.

Scheerer, S. (1978). Der politisch-publizistische Verstärkerkreislauf. Zur Beeinflussung der Massenmedien im Prozeß strafrechtlicher Normgenese. Kriminologisches Journal, 10, 223-227.

Schetsche, M. (2014). Empirische Analyse sozialer Probleme. Wiesbaden: Springer VS.

Smith, D. A. (1986). The neighborhood context of police behavior. Crime and Justice, 8, 313-341.

Stadt Essen (2020). Ein Blick auf .... Stadtteile in Essen Altendorf 2019. URL: https://media.essen.de/ media/wwwessende/aemter/12/ein_blick_auf_stadtteile/Altendorf_07.pdf. Zugegriffen: Juni 2021.

Stadt Essen (1999). Handlungskonzept für Essen-Altendorf-Stadtteil mit besonderem Erneuerungsbedarf. Essen: o.A.

Stehr, J. (1998). Sagenhafter Alltag. Über die private Aneignung herrschender Moral. Frankfurt a. M.: Campus.

Steinert, H. (1998). Genau hinsehen, geduldig nachdenken und sich nicht dumm machen lassen. In H. Steinert (Hrsg.), Zur Kritik der empirischen Sozialforschung. Ein Methodengrundkurs (S. 67-79). Frankfurt a. M.: Johann Wolfgang von Goethe Universität.

Terrill, W., \& Reisig, M. D. (2003). Neighborhood context and police use of force. Journal of Research in Crime and Delinquency, 40, 291-321.

Thomas, W. I., \& Thomas, D. S. (1928). The child in America. Behavior problems and programs. New York: A. A. Knopf.

Thompson, V. E. (2018). „There is no justice, there is just us!“": Ansätze zu einer postkolonial-feministischen Kritik der Polizei am Beispiel von Racial Profiling. In D. Loick (Hrsg.), Kritik der Polizei (S. 197-219). Frankfurt a. M.: Campus.

Volkmann, A. (2012). Quartierseffekte in der Stadtforschung und in der sozialen Stadtpolitik. Berlin: Universitätsverlag der Technischen Universität Berlin.

Wacquant, L. (1996). Red belt, black belt: Racial division, class inequality and the state in French urban periphery and the American ghetto. In E. Minigione (Hrsg.), Urban poverty and the underclass (S. 234-274). Oxford (UK), Cambridge (USA): Blackwell Publishers. 
Wacquant, L. (2018). Die Verdammten der Stadt. Interkulturelle Studien. Wiesbaden: Springer.

Wacquant, L., Slater, T., \& Pereira, V. B. (2014). Territorial stigmatization in action. Evironment and Planning A, 46, 1270-1280.

Waddington, P. A. J., Stenson, K., \& Don, D. (2004). In proportion. Race, and police stop and search. British Journal of Criminology, 44, 889-914.

Walther, U.-J., \& Güntner, S. (2013). Aufstieg und Fall der sozialen Stadtpolitik in Europa - Das Ende einer Ära? In M. Kronauer \& W. Siebel (Hrsg.), Polarisierte Städte. Soziale Ungleichheit als Herausforderung für die Stadtpolitik (S. 287-308). Frankfurt a. M.: Campus.

WAZ (Westdeutsche Allgemeine Zeitung) (2019). Arabische Hochzeitsfeier löst Großeinsatz der Polizei aus. WAZ.de vom 6. Aug. 2019. URL: https://www.waz.de/staedte/essen/arabische-hochzeitsfeierloest-grosseinsatz-der-polizei-aus-id226702577.html. Zugegriffen: Juni 2021.

Wegner, M., Wagner, D., vom Feld, L., \& Struck, J. (2020). Die Polizei als ,Influencerin“? - Zum Einfluss der Polizei auf sicherheitspolitische Diskurse: Die Silvesternacht in Leipzig-Connewitz 2019/2020. In D. Hunold \& A. Ruch (Hrsg.), Polizeiarbeit zwischen Praxishandeln und Rechtsordnung (S. 39-76). Wiesbaden: Springer VS.

Wehrheim, J. (2012) [2002]. Die überwachte Stadt. Sicherheit, Segregation und Ausgrenzung. 3. Aufl. Opladen u.a.: Barbara Budrich.

Weuster, K. (2019). Polizei erschießt Messer-Mann in Essen. BILD.de vom 19. Juni 2019. URL: https://www.bild.de/regional/ruhrgebiet/ruhrgebiet-aktuell/angreifer-wollte-wohl-sterben-polizisterschiesst-messer-mann-in-essen-62699328.bild.html. Zugegriffen: Juni 2021.

Wilson, J. Q., \& Kelling, G. L. (1982). Broken Windows. Atlantic Monthly, 249, 29-38.

Wiest, K., \& Kirndörfer, E. (2019). Paradoxe Aushandlungen von Migration im Diskurs um die Leipziger Eisenbahnstraße. Raumforschung und Raumordnung, 77, 1-18.

\section{Zitierte Interviews und Erhebungsprotokolle}

Bew_Adorf_B_08. Teilstrukturiertes Interview, Bewohnerin, ca. 50 Jahre, Oktober 2018.

Bew_Adorf_D_02. Teilstrukturiertes Interview, drei Bewohner, ca. 25, 30 und 40 Jahre, September 2018. Bew_Adorf_D_13. Teilstrukturiertes Interview, Bewohner, ca. Ende 20 Jahre, Oktober 2018.

Bew_Adorf_D_10. Teilstrukturiertes Interview, Bewohner, ca. 60 Jahre, Oktober 2018.

Bew_Adorf_D_20. Teilstrukturiertes Interview, zwei Bewohner, ca. 16 und 18 Jahre, Oktober 2018.

Na_Adorf_05_D. Narratives Interview, Bewohnerin, ca. 25 Jahre, April 2019.

Exp_Adord_01. Expert*inneninterview, zwei Mitarbeiter*innen der Stadtteilarbeit, Juli 2018.

Exp_Adorf_04. Expert*inneninterview, drei Mitarbeiter*innen Stadt Essen, September 2018.

Exp_Adorf_05. Experteninterview, Mitarbeiter Kreispolizeibehörde Essen, September 2018.

Erhebungsprotokoll Anbahnung narrativ-episodale Interviews Altendorf, 18.4.2019_D.

Feldnotizen Altendorf, 15. 6. 2018_D.

Moritz Rinn geb. 1982. Dr. phil., Wissenschaftlicher Mitarbeiter am Institut für Soziale Arbeit und Sozialpolitik der Universität Duisburg-Essen. Forschungsschwerpunkte: Städtische Konflikte und Transformationen, Politiken des Sozialen. Aktuelle Veröffentlichungen: (mit L. Wiese) Politiken sozialer Mischung und die Produktivität von Rassismus im ,gefährlichen Viertel“, in: Geographica Helvetica, 2020; Ein Urbanismus der Ungleichheit, in: sublurban 2018; Konflikte um die Stadt für alle, 2016.

Jan Wehrheim geb. 1967. Dr. rer. pol., Professor für Soziologie am Institut für Soziale Arbeit und Sozialpolitik der Universität Duisburg-Essen. Forschungsschwerpunkte: Stadtsoziologie, Soziologie sozialer Kontrolle, Techniksoziologie/Surveillance Studies. Aktuelle Veröffentlichungen: (Hrsg.) Sanfte Kontrolle? Devianz, Etikettierung und Soziale Arbeit: 1975 und 2020, 2021; (Hrsg. m. N. Gestring) Urbanität im 21. Jahrhundert, 2018; Die überwachte Stadt (3. Aufl.), 2012. 